\title{
CARACTERIZACIÓN SOCIODEMOGRÁFICA DE ESTUDIANTES QUE RINDEN LA PSU, POSTULAN Y SE MATRICULAN EN UNIVERSIDADES DEL CONSEJO DE RECTORES
}

\section{Antecedentes}

El Consejo Superior de Educación (CSE) se planteó el desafío de profundizar el estudio y análisis de temas atingentes a la educación terciaria, con el fin de propiciar el desarrollo de competencias institucionales que permitan promover el debate nacional en torno a la materia. En esa línea, se planteó que una tarea prioritaria era determinar si el país contaba -o contará dentro de un plazo razonable-con un sistema de educación postsecundaria que forme los profesionales y técnicos que, por su cantidad y su calidad, permitan alcanzar efectivamente las metas de desarrollo, equidad, bienestar y calidad de vida, acordes a los requerimientos de crecimiento, dinamismo económico e incertidumbre, propios de la globalización.

Al respecto, cabe mencionar los esfuerzos por incrementar la cobertura en todos los sistemas, los que han posicionado a la educación como uno de los medios más importantes para determinar, por una parte, el desarrollo de una nación y, por otra, favorecer la movilidad social. Es así como la cobertura en educación básica supera el 95\% (Belleï, 2003) y la de educación media ha aumentado entre 1977 y 2002 en 10 puntos porcentuales (de 77\% a 87\%) (Lemaitre, 2003). Asimismo, en los resultados de la encuesta CASEN (2003) se determinó que la cobertura en educación superior ha crecido más del doble en los últimos 13 años (de un 16\% a un $37,5 \%)$.

Este creciente acceso a la educación superior presenta diferencias entre los distintos grupos socioeconómicos. Entre 1990 
y 2003 el incremento más notorio se produjo en los hogares de mayores ingresos económicos. Sin perjuicio de lo anterior, resulta interesante destacar que en el $40 \%$ de los hogares de menores ingresos (quintil I y II) el aumento se triplicó durante el periodo analizado (pasando de $4,4 \%$ a $14,5 \%$ en el quintil I, y de $7,8 \%$ a 21,2\% en el segundo quintil) (CASEN, 2003).

Este aumento de la cobertura ha traído aparejado una ampliación de la oferta académica, que se refleja en el surgimiento tanto de un creciente y progresivo número de instituciones de educación terciaria, como también de una mayor cantidad de carreras ofrecidas. Al respecto, existen a lo menos cuatro grupos de instituciones que acogen estudiantes postsecundarios:

1. Universidades (públicas y privadas).

2. Institutos profesionales.

3. Centros de formación técnica (públicos y privados).

4. Fuerzas Armadas (FF.AA.) y de Orden (Carabineros de Chile y Policía de Investigaciones).

En conjunto, estas instituciones poseen una matrícula de 527.960 alumnos, los que pueden diferenciarse en 432.999 de carreras universitarias, 84.935 de carreras técnicas y 10.026 de centros de educación pertenecientes a las FF.AA. y de Orden ${ }^{\text {ii }}$.

Sin embargo, los datos recién expuestos no dan cuenta de las características de los estudiantes que se matriculan en alguna de estas instituciones. Por ello, es necesario analizar, primero, sus características (específicamente, sociodemográficas y educacionales) para luego determinar su posible incidencia en la elección de alguna de las instituciones de educación superior. La principal hipótesis postula que un elemento determinante de la matrícula se encuentra en las variables socioeconómicas y culturales propias de cada estudiante.

ii Base estadística Indicadores, Números y Datos sobre Instituciones y Carreras de Educación Superior (INDICES, CSE) 2004. 
La conjetura se basa en un conjunto de teorías y supuestos surgidos desde la psicología social, específicamente de los aportes de la teoría de los grupos humanos (Shaw, 1986; Brown, 1997) y de las relaciones intergrupales en lo referido a la identidad social (Tajfel, 1984; Morales; Páez; Deschamps y Worchel, 1996). Estas últimas plantean, principalmente, que las personas se congregan en grupos que les dan identidad y que dichos grupos presentan diferencias de estatus sociales; asimismo, la relación entre los grupos se da a partir de esas diferencias de estatus (Tajfel, 1984; Fiske, 2000). Ello trae como consecuencia el surgimiento de un conjunto de estereotipos y prejuicios respecto del grupo propio y de los otros grupos (Brown, 1997; Morales; Páez; Deschamps y Worchel, 1996), los que podrían verse influenciados por factores socioeconómicos y educacionales.

El objetivo de la presente investigación es describir las características sociodemográficas y educacionales de los estudiantes que se matriculan en alguna de las universidades del CRUCH. En forma subsidiaria al objetivo recién planteado, se intentará establecer si existen determinadas características comunes y distintivas entre los alumnos que se matriculan en cada universidad.

\section{Metodología}

La presente investigación corresponde a un estudio de tipo descriptivo-relacional, ya que el propósito es describir a los estudiantes en términos de sus características educacionales y sociodemográficas, intentando determinar si alguna de ellas se vincula con la elección de un determinado centro de educación superior. Asimismo, y debido a que no existe una manipulación intencionada de variables (sino sólo la medición de diversas características), el diseño de investigación es no experimental (o ex pos facto).

La población estuvo compuesta por los 28.906 estudiantes matriculados en alguna universidad del $\mathrm{CRUCH}$, que egresaron de 
establecimientos educacionales en el año 2005 y rindieron la Prueba de Selección Universitaria (PSU) ese mismo año. Para lograr un análisis más detallado del proceso conducente a la matrícula fue necesario incluir al total de alumnos que rindió la prueba PSU, postuló a alguna de las universidades del CRUCH y, finalmente, se matriculó en una de ellas. De este modo, la población en estudio queda definida de la siguiente manera:

\begin{tabular}{lc}
\hline Proceso & Cantidad de estudiantes \\
\hline Rendición de PSU & 122.014 \\
Postulación & 51.811 \\
Matrículas en universidades del CRUCH & 28.906 \\
\hline
\end{tabular}

Todos los datos se recolectaron y sistematizaron a partir de la base de datos entregada por DEMRE y fueron analizados con el apoyo del programa estadístico "SPSS", versión 13.0.

\section{Resultados}

El proceso conducente al ingreso de un estudiante en alguna de las 25 universidades adscritas al CRUCH se refleja en el siguiente gráfico:

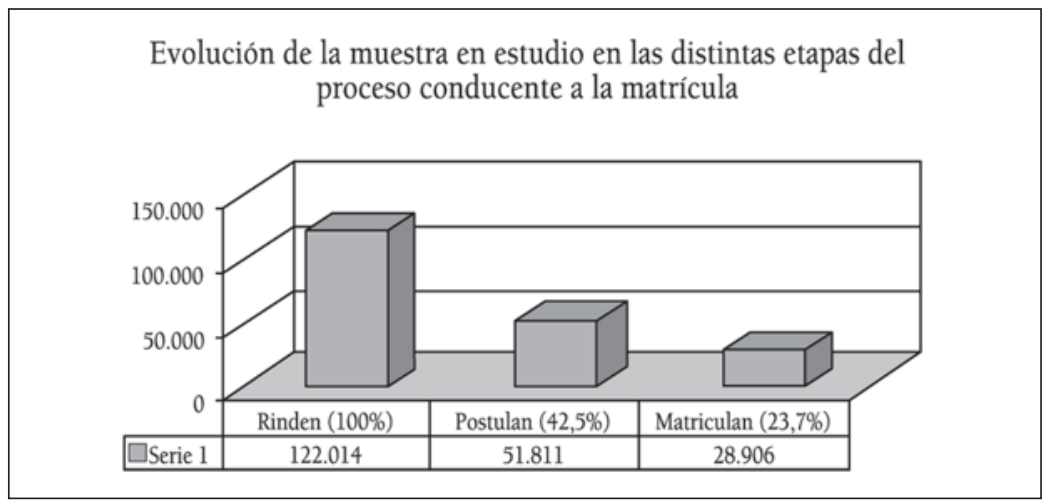

De este modo, de los 122.014 egresados de establecimientos educacionales en 2005 que rindieron la prueba PSU, sólo el 42,5\% $(\mathrm{N}=51.811)$ postula a estas instituciones. Esta disminución se acentúa en la última parte del proceso, ya que solamente 28.906 estudiantes se matriculan (lo que equivale al $23,7 \%$ de la población que rindió la PSU). En otras palabras, cerca de 100.000 jóvenes 
quedan marginados o se marginan voluntariamente del proceso conducente a la matrícula en una universidad del Consejo de Rectores ${ }^{\mathrm{iii}}$.

Otro elemento llamativo radica en que quienes se matriculan en las universidades del CRUCH comparten determinadas características sociodemográficas y educacionales:

- Provienen de establecimientos científico-humanistas.

- Estudiaron en colegios subvencionados (particulares o municipales).

- Sus padres presentan altos niveles educacionales y un trabajo bien remunerado.

- Son mayoritariamente varones.

Considerando la disminución ya señalada y estas características, resultó pertinente establecer si las variables sociodemográficas y educacionales se asocian con la matrícula de determinado alumno. Para lograr este cometido, fue necesario considerar las características de los estudiantes que participaron en cada una de las tres etapas conducentes a la inserción del alumnado en una de las universidades del CRUCH (rendición PSU, postulación y matrícula).

De este modo, la presentación de los resultados se realizará segmentada en tres acápites. El primero de ellos se aboca a describir las características sociodemográfica de los estudiantes que participan en cada fase del proceso. Posteriormente, se desarrolla una descripción exclusiva de los estudiantes que decidieron no postular a alguna universidad del CRUCH, aun cuando alcanzaron un puntaje suficiente como para hacerlo. Finalmente, se presenta un análisis comparativo de las universidades pertenecientes al Consejo de Rectores.

iii Se debe recordar que este análisis se basa solamente en aquellos estudiantes que dieron la PSU por primera vez el año 2005. Para ese año hubo alrededor de 50.000 estudiantes que rindieron la PSU por segunda o más veces; ellos no están integrados en este análisis. 


\section{Caracterización sociodemográfica y educacional de los estudiantes que rinden la PSU, postulan y se matriculan en alguna universidad del CRUCH}

Tal como se enunció al inicio de este documento, la caracterización se llevó a cabo considerando las variables incluidas en la base de datos entregadas por DEMRE. Así, al analizar las tres etapas en función de la rama educacional, se observa que los estudiantes provenientes de establecimientos científico-humanistas aumentan proporcionalmente en cada una de las etapas descritas. Lo anterior se puede apreciar en los datos de la siguiente tabla:

\begin{tabular}{lrrrrrr}
\hline & \multicolumn{2}{c}{ Rinden } & \multicolumn{2}{c}{ Postulan } & \multicolumn{2}{c}{ Matriculan } \\
\hline Científico-humanista & 95.000 & $(78 \%)$ & 46.204 & $(89 \%)$ & 26.112 & $(90 \%)$ \\
Técnico-profesional & 27.014 & $(22 \%)$ & 5.607 & $(11 \%)$ & 2.794 & $(10 \%)$ \\
Total & 122.014 & $(100 \%)$ & 51.811 & $(100 \%)$ & 28.906 & $(100 \%)$ \\
\hline
\end{tabular}

El aumento de los estudiantes provenientes de la rama científico-humanista se asocia con un decremento porcentual de los egresados de colegios técnico-profesionales, quedando matriculados finalmente menos de la mitad de su porcentaje inicial. En efecto, si bien al momento de rendir la PSU este subgrupo correspondía al $22 \%$ del conjunto ( $\mathrm{N}=27.014)$, al momento de matricularse se reduce al 10\% $(\mathrm{N}=2.794)$.

Ahora, si se analiza la dependencia administrativa del establecimiento de egreso de los jóvenes al momento de rendir la prueba, los colegios particulares subvencionados y municipales alcanzan un porcentaje similar de estudiantes (44\% y $41 \%$ respectivamente). No obstante, al momento de matricularse hay una disminución de los colegios municipales, lo que se refleja en la siguiente tabla:

\begin{tabular}{lllllll}
\hline & \multicolumn{2}{c}{ Rinden } & \multicolumn{2}{c}{ Postulan } & \multicolumn{2}{c}{ Matriculan } \\
\hline Particular pagado & 18.692 & $(15 \%)$ & 11.574 & $(22 \%)$ & 6.376 & $(22 \%)$ \\
Particular subvencionado & 53.128 & $(44 \%)$ & 22.540 & $(44 \%)$ & 12.422 & $(43 \%)$ \\
Municipal & 50.194 & $(41 \%)$ & 17.697 & $(34 \%)$ & 10.108 & $(35 \%)$ \\
\hline
\end{tabular}


A su vez, los estudiantes provenientes de colegios particulares subvencionados mantienen una proporción similar en cada uno de los tres momentos. Por último, los estudiantes que aumentan su representación a lo largo del proceso son aquellos que provienen de establecimientos particulares pagados; en otras palabras, si bien los estudiantes provenientes de estas dependencias administrativas representan el grupo de menor proporción al interior del conjunto, es el único subgrupo que aumenta su representación a lo largo del proceso.

En lo referente al nivel educacional de los padres, la proporción de estudiantes cuyos padres alcanzan niveles educacionales universitarios aumenta desde el momento de rendición de PSU hasta la matrícula, en desmedro de los estudiantes cuyos padres poseen niveles inferiores de educación (básica completa, incompleta o sin estudios). Los hijos de padres con niveles básicos de educación pierden participación en cada una de las etapas del proceso ya descrito.

Una situación semejante ocurre con los ingresos familiares reportados por los estudiantes al momento de rendir la PSU. Aquellos que reportan ingresos inferiores a \$278.000 disminuyen un $9 \%$ entre la etapa de rendición de la PSU y la de matrícula, situación que se repite con los estudiantes cuyas familias generan ingresos que fluctúan entre \$27.8001 a \$ 83.4000 (se aprecia un aumento porcentual de $5 \%$ ). Todo lo ya referido queda reflejado en la siguiente tabla:

\begin{tabular}{|c|c|c|c|c|c|c|c|}
\hline Ingreso far & miliar autorreportado & Rin & den & Post & ulan & Matric & ulan \\
\hline Menos de & $\$ 278.000$ & 67.753 & $(56 \%)$ & 23.072 & $(45 \%)$ & 12.878 & $(45 \%)$ \\
\hline & $\$ 278.001$ a $\$ 834.000$. & 36.567 & $(30 \%)$ & 17.934 & $(35 \%)$ & 10.008 & $(35 \%)$ \\
\hline & $\$ 834.001$ a $\$ 1.400 .000$ & 8.775 & $(7 \%)$ & 5.250 & $(10 \%)$ & 3.008 & $(10 \%)$ \\
\hline & $\$ 1.400 .001$ a $\$ 1.950 .000$. & 3.356 & $(3 \%)$ & 2.149 & $(4 \%)$ & 1.189 & $(4 \%)$ \\
\hline & $\$ 1.950 .00$ a $\$ 2.500 .00$ & 2.061 & $(2 \%)$ & 1.337 & $(3 \%)$ & 717 & $(2 \%)$ \\
\hline Más de & $\$ 2.500 .00$ & 3.142 & $(3 \%)$ & 2.064 & $(4 \%)$ & 1.105 & $(4 \%)$ \\
\hline Total & & 122.014 & $(100 \%)$ & 51.811 & (100\%) & 28.906 & $(100 \%)$ \\
\hline
\end{tabular}


En cuanto a las restantes categorías de ingreso familiar, aun cuando numéricamente son más bajas en el proceso de rendición de PSU, porcentualmente muestran una leve tendencia al aumento en las dos siguientes etapas.

Se observa que la representación entre sexos varía en cada etapa, partiendo desde una situación de mayor frecuencia del sexo femenino (etapa de rendición, con 53\%) hacia una en la que la proporción se invierte a favor del sexo masculino, el que termina ocupando el 52\% del total (en la matrícula). Ello se puede apreciar en la tabla a continuación:

\begin{tabular}{lrrrrrr}
\hline & \multicolumn{2}{c}{ Rinden } & \multicolumn{2}{c}{ Postulan } & \multicolumn{2}{c}{ Matriculan } \\
\hline Femenino & 64.349 & $(53 \%)$ & 26.366 & $(51 \%)$ & 13.982 & $(48 \%)$ \\
Masculino & 57.665 & $(47 \%)$ & 25.445 & $(49 \%)$ & 14.924 & $(52 \%)$ \\
Total & 122.014 & $(100 \%)$ & 51.811 & $(100 \%)$ & 28.906 & $(100 \%)$ \\
\hline
\end{tabular}

Así, de los 57.665 hombres y 64.349 mujeres que rinden la prueba, un $41 \%$ de las mujeres $(\mathrm{N}=26.366)$ pasa a la etapa de postulación, mientras que un $44 \%(\mathrm{~N}=25.445)$ en el caso de los hombres. Finalmente, al pasar a la etapa de matrícula se observa que el 22\% ( $\mathrm{N}=13.982)$ de la población inicial de mujeres se matricula, porcentaje que se eleva al 26\% ( $\mathrm{N}=14.924)$ en el caso de los hombres. Este hecho llama la atención en la medida en que no parece fácil explicar el motivo de la disminución proporcional de las mujeres en relación con los hombres.

Al analizar la distribución de los puntajes obtenidos en la PSU, se observa que el 70, 5\% de los alumnos que rinden la prueba obtiene puntajes inferiores a 550 y que sólo un $7,5 \%$ de ellos (alrededor de 16.300 estudiantes) obtiene puntajes por sobre 650 puntos.

Desde el punto de vista de la distribución regional, se observa que las regiones Metropolitana y XI obtienen los mayores valores promedio (500), mientras que las regiones I, III y IV alcanzan los 
valores más bajos (475 puntos en promedio). Al respecto, es prudente recordar que el puntaje promedio total es de 492,1 puntos (con una desviación estándar de 105,4), lo que significa que las regiones Metropolitana, VII, X, XI y XII se encuentran sobre la media nacional.

Al analizar los puntajes promedio según rama educacional se observa una notoria brecha (131 puntos) entre la modalidad que obtiene valores superiores (científico-humanista diurno con 516,1 puntos) y la modalidad que obtiene valores inferiores (técnico-profesional agrícola con 385,1). Junto con ello, se observa una variación de más de cien puntos entre la jornada diurna y nocturna de la modalidad científico-humanista.

En lo relativo a la dependencia de los establecimientos educacionales de proveniencia, ésta se encuentra asociada a importantes diferencias en los puntajes de la PSU. Concretamente, los establecimientos particulares pagados (que obtienen un promedio de 581,1 puntos) obtienen 118,5 puntos más que los establecimientos municipales (promedio de 462,6) y 93,2 puntos por sobre los particulares subvencionados (de 487,9 puntos). Por su parte, la diferencia entre estos últimos y los municipales es de 25,3 puntos.

En síntesis, las características más relevantes del grupo estudiado son las siguientes:

- Se observa una asociación directa entre ingresos familiares y puntajes en la PSU; el grupo que reporta ingresos más bajos obtiene sólo un promedio de 459,6 puntos, mientras que el que reporta ingresos más altos, obtiene un promedio de 619,5, lo que significa una diferencia de 159,9 puntos.

- El nivel educacional de ambos padres se encuentra correlacionado positivamente con los puntajes PSU. De acuerdo con lo anterior, se observa que los puntajes crecen al mismo tiempo que aumentan los niveles educacionales de ambos padres. Se aprecia una importante diferencia entre los 
estudiantes cuyos padres reportan educación básica incompleta y aquellos que reportan educación universitaria completa.

- La situación laboral de los padres tiende a marcar diferencias entre quienes rinden la PSU; quienes tienen padres activos laboralmente obtienen puntajes superiores a aquellos cuyos padres se encuentran cesantes, con trabajos ocasionales o no trabajan.

- Se observa una asociación entre el cargo desempeñado por los padres y los puntajes PSU obtenidos por sus hijos, en tanto quienes obtienen mayores puntajes son aquellos cuyos padres se desempeñan como gerentes o son profesionales de carreras de cinco o más años. Por otra parte, aquellos que obtienen puntajes más bajos tienen padres que se desempeñan como obreros agrícolas calificados o no calificados.

\section{Caracterización sociodemográfica y educacional de los estudiantes que no postulan a alguna universidad del CRUCH}

Al analizar las características de los estudiantes que no postulan a una universidad del Consejo de Rectores, aun cuando poseen puntajes superiores a 450, se observan características educacionales y sociodemográficas diferentes de aquellos que sí lo hacen. Existe una diferencia de puntaje PSU de 64,7 puntos a favor de los alumnos que sí postulan; sin embargo, este último grupo obtiene un puntaje promedio superior a 500 puntos (puntuación con la que hubieran sido aceptados en alguna de las carreras impartidas por estas universidades).

Provienen proporcionalmente en mayor cantidad de establecimientos técnico-profesionales y municipales; así, el total de alumnos que no postulan está compuesto por un $78 \%$ de jóvenes egresados de colegio científico-humanistas (HC) y un $22 \%$ salido de colegios técnico-profesionales (TP). Lo anterior implica que estos últimos duplican su participación porcentual en el grupo de los no 
postulados en relación con el grupo de los postulados (donde ocupan un $11 \%$ del conjunto). Al respecto, es posible concluir que el proceso de postulación capta mayoritariamente a los egresados de establecimientos HC, mientras que los egresados de colegio TP -una vez rendida la PSU- disminuyen su participación en el proceso conducente a la matrícula y engrosan su participación en los grupos de los no postulados. Dicho de otro modo, los egresados de colegios TP tienen, finalmente, menos posibilidades de ingresar a una universidad del CRUCH.

Se observa que el proceso de postulación segrega a alumnos egresados principalmente de establecimientos municipales, en beneficio de los provenientes de colegios particulares pagados. La principal diferencia en la distribución de casos se presenta al comparar los establecimientos municipales con los particulares pagados. Si bien en cuanto a la frecuencia total, los colegios municipales "aportan" una mayor cantidad de jóvenes al proceso de postulación que los particulares pagados, no es menos cierto que el mayor porcentaje de los egresados de este tipo de establecimiento no postula. La situación contraria se encuentra en los particulares pagados, donde la mayoría de sus alumnos postula a alguna universidad. Por último, no se aprecian diferencias en los colegios particulares subvencionados.

En relación con las características familiares de los alumnos que no postulan a la universidad, se determina que sus padres tienen niveles educacionales más bajos, equivalente a enseñanza media o inferior (sumando todos los casos, se detecta que más del 60\% se encuentra en esta situación), mientras que casi la mitad de los estudiantes que sí postulan reportan tener padres con un nivel educativo técnico o universitario (aglutinando ambas categorías, casi el 47\%). Aún más, la diferencia más evidente está en el nivel educacional universitario, donde la distancia entre alumnos que postulan de aquéllos que no lo hacen es de $8 \%$ (36,5\% v/s 28,3\%). Es posible concluir que la presencia de padres con niveles de educación superior es mayor en las personas que deciden postular 
a alguna universidad. Al respecto, los que no postulan tienen un mayor porcentaje de madres con menores niveles de escolaridad (media y menos) y los que sí lo hacen tienen madres con un mayor porcentaje de estudios superiores.

\section{Análisis comparativo de las 25 universidades adscritas al CRUCH, a partir de las variables de caracterización educacionales y sociodemográficas}

A continuación se presenta un análisis descriptivo-comparativo de las principales características de los estudiantes que postulan y se matriculan en las diferentes universidades del CRUCH. Para ello, se han elaborado trece capítulos que incorporan la información para cada variable en estudio en todas las universidades. Las tablas correspondientes registran a las universidades en orden descendente, en función del atributo estudiado. De esta manera, aquellas universidades que presentan en mayor medida la variable evaluada han quedado en los primeros lugares y, por el contrario, la baja presencia de dicha variable se asocia a una posición menor (siendo la más baja el lugar 25). Finalmente, toda la información recopilada se sistematiza en un índice, que refleja el nivel de preferencia alcanzado por cada universidad por parte de los estudiantes egresados del colegio en el año 2005.

\section{III.1. Proceso de postulación}

Este proceso refleja los intereses de los estudiantes antes de matricularse; señala cómo son valoradas las instituciones de educación superior con relación a una serie de variables.

Aunque quienes postulan a la universidad tienen ocho opciones, ha parecido conveniente observar el comportamiento de las tres primeras preferencias, en la medida en que estas muestran aquellas que los futuros estudiantes más ambicionan. Este hecho también queda reflejado en la cantidad de personas que es posible contabilizar en cada postulación: para la primera preferencia se 
encuentran 51.811 postulaciones; para la segunda, 49.235, y para la tercera, 45.667; en contraste con lo anterior, en las postulaciones posteriores -cuarta a octava- la frecuencia total desciende notablemente, presentándose finalmente sólo 12.337 postulaciones en la octava preferencia.

Ahora, para desarrollar este análisis, junto con informar el porcentaje final de postulaciones que acumula cada institución (sumando las tres primeras preferencias) se ha decidido reportar el puntaje PSU que promedian los postulantes a estas casas de estudio, lo que se refleja en la tabla siguiente:

\begin{tabular}{clcc}
\hline Lugar & UNIVERSIDAD & $\begin{array}{c}\text { Porcentaje considerando } \\
\text { 3 primeras postulaciones* }\end{array}$ & $\begin{array}{c}\text { Puntaje PSU } \\
\text { promedio* }\end{array}$ \\
\hline 1 & De Chile & 12,30 & 643,5 \\
2 & De Concepción & 12,06 & 566,1 \\
3 & Pontificia Univ. Católica de Chile & 9,05 & 652,3 \\
4 & De Valparaíso & 7,41 & 554,9 \\
5 & De Santiago & 7,22 & 582,0 \\
6 & Pontificia Univ. Católica de Valparaíso & 7,15 & 565,4 \\
7 & Téc. Federico Sta. María & 5,73 & 585,7 \\
8 & De La Frontera & 3,89 & 549,5 \\
9 & De La Serena & 3,63 & 520,6 \\
10 & Austral & 3,39 & 562,3 \\
11 & De Playa Ancha & 2,72 & 519,6 \\
12 & Del Bío-Bío & 2,72 & 532,3 \\
13 & Católica del Norte & 2,64 & 550,7 \\
14 & De Talca & 2,48 & 566,1 \\
15 & De Tarapacá & 2,11 & 513,4 \\
16 & Metropolitana de Ciencias de la Educ. & 2,10 & 548,4 \\
17 & Católica de la Santísima Concepción & 1,85 & 541,0 \\
18 & De Antofagasta & 1,85 & 529,7 \\
19 & Católica de Temuco & 1,75 & 518,1 \\
20 & Arturo Prat & 1,70 & 505,0 \\
21 & Tecnológica Metropolitana & 1,67 & 562,3 \\
22 & Católica del Maule & 1,58 & 538,5 \\
23 & De Los Lagos & 1,45 & 504,9 \\
24 & De Magallanes & 0,84 & 518,1 \\
25 & De Atacama & 0,71 & 506,0 \\
\hline & Totales & 100,00 & 549,5 \\
\hline & & & \\
\hline
\end{tabular}

* Tanto los porcentajes como el promedio de puntaje en la PSU se obtuvieron ponderando los valores obtenidos en las tres primeras postulaciones. 
Tal como se desprende de la tabla precedente, existe una preferencia marcada por postular a algunas universidades; es más, las tres primeras opciones de postulación (universidades de Chile (UCH), de Concepción (UDC) y Pontificia Universidad Católica de Chile (PUC)) concentran alrededor de un tercio de todas las postulaciones y tienden, a su vez, a concentrar altos puntajes en aquellas tres primeras preferencias. Por el contrario, aquellas instituciones que acapararon un muy bajo porcentaje de postulaciones (universidades de Los Lagos, de Magallanes y de Atacama) poseen potenciales estudiantes de un promedio PSU levemente superior a los 500 puntos.

Debido a lo ya señalado, resultó pertinente determinar si efectivamente existía una asociación entre el porcentaje de personas que postulan a una casa de estudio específica y el puntaje PSU con que lo hacen. Para resolver dicha incógnita se utilizó el procedimiento de correlación Rho de Spearman. Se logró establecer que existe una correlación positiva y significativa entre las dos variables ( $r s=0,754 ; p<0.001$ ). La asociación indica que las universidades que poseen una mayor cantidad de postulantes (en primera, segunda o tercera preferencia) concentran los mejores puntajes promedio PSU. De este modo, se observa que las siete primeras universidades de la tabla (ubicadas en las regiones V, VIII y Metropolitana) aglutinan el 60,92\% de las postulaciones con un promedio PSU de 592,8. Ello significa que las 18 restantes se reparten alrededor de un $40 \%$ de las postulaciones (con un promedio de 532,6 puntos).

Resulta importante destacar que nueve universidades no reciben una frecuencia de postulaciones en sus primeras tres preferencias superiores al 2\% (en la tabla, desde la Universidad Católica de la Santísima Concepción hacia abajo), lo que permite afirmar que no son priorizadas por los futuros estudiantes. 


\section{III.2. Matrícula por universidad}

La información contenida en la tabla siguiente muestra la frecuencia y el porcentaje de matrícula de cada universidad, así como el puntaje PSU promedio asociado a dicha matrícula:

\begin{tabular}{clrrc}
\hline Lugar & UNIVERSIDAD & Frecuencia & Porcentaje & $\begin{array}{c}\text { Puntaje PSU } \\
\text { promedio }\end{array}$ \\
\hline 1 & De Concepción & 2.988 & 10,34 & 606,1 \\
2 & De Chile & 2.779 & 9,61 & 686,8 \\
3 & Pontificia Univ. Católica de Chile & 2.394 & 8,28 & 694,2 \\
4 & De Santiago & 1.956 & 6,77 & 614,7 \\
5 & De Valparaíso & 1.855 & 6,42 & 583,4 \\
6 & Pontifica Univ. Católica de Valparaíso & 1.759 & 6,09 & 608,3 \\
7 & Téc. Federico Sta. María & 1.682 & 5,82 & 616,7 \\
8 & Austral & 1.241 & 4,29 & 581,6 \\
9 & Del Bío-Bío & 1.129 & 3,91 & 551,6 \\
10 & Católica del Norte & 1.118 & 3,87 & 573,4 \\
11 & De La Serena & 987 & 3,41 & 553,9 \\
12 & De La Frontera & 890 & 3,08 & 594,8 \\
13 & De Tarapacá & 852 & 2,95 & 532,1 \\
14 & Católica de Temuco & 826 & 2,86 & 534,6 \\
15 & De Talca & 786 & 2,72 & 590,1 \\
16 & Tecnológica Metropolitana & 767 & 2,65 & 588,9 \\
17 & De Playa Ancha & 742 & 2,57 & 550,0 \\
18 & De Antofagasta & 710 & 2,46 & 547,9 \\
19 & Católica de la Santísima Concepción & 677 & 2,34 & 551,5 \\
20 & Arturo Prat & 643 & 2,22 & 526,2 \\
21 & De Los Lagos & 541 & 1,87 & 524,4 \\
22 & Católica del Maule & 476 & 1,65 & 569,1 \\
23 & Metropolitana de Ciencias de la Educ. & 408 & 1,41 & 593,5 \\
24 & De Magallanes & 378 & 1,31 & 537,8 \\
25 & De Atacama & 322 & 1,11 & 525,6 \\
\hline & Total & 28.906 & 100 & 577,5 \\
\hline & & & & \\
\hline
\end{tabular}

Se desprende de estos datos, que más de la mitad de las matrículas $(53,32 \%)$ se concentran en siete universidades. Las otras 18 universidades acumulan el 46,68\% restante.

También, cinco universidades no alcanzan al 2\% de la matrícula, y tres de ellas están ubicadas en regiones con importantes grupos poblacionales (situación válida para la Universidad Metropolitana de Ciencias de la Educación (UMCE), Universidad Católica del Maule (UCM) y Universidad de Los Lagos). Ello 
significa que un número importante de estudiantes no tienen a estas universidades como primera opción. Ello puede deberse al tamaño de la universidad, al tipo de carreras que ofrece y/o a que la calidad no les satisface.

Las siete instituciones con mayor matrícula obtienen alumnos con 630 puntos promedio en la PSU, mientras que las 18 restantes (cuya matrícula acumula un 46,7\%) reclutan alumnos que exhiben un puntaje promedio de 581,6 (inferior en casi 50 puntos). Sin embargo, ambos valores son superiores a los obtenidos en el proceso de postulación.

\section{III.3. Puntajes PSU y matrículas}

El análisis del puntaje PSU promedio alcanzado por los estudiantes matriculados muestra que dichos valores fluctúan de manera importante al compararlos entre las universidades. Sólo seis instituciones (PUC, UCH, Universidad Técnica Federico Santa María (UTFSM), Universidad de Santiago (USACH), Universidad Católica de Valparaíso (UCV) y UDC) incorporan estudiantes cuyo puntaje promedio es superior a los 600 puntos $(637,8)$. Al respecto, cabe señalar que dos de ellas alcanzan puntajes significativamente superiores al resto (PUC y UCH). En cuanto a las 19 restantes, éstas se ubican bajo los 600 puntos (promedio $=558,5$ ).

A continuación se presenta una tabla en la que se pueden apreciar los puntajes PSU promedio que alcanzan los matriculados a cada una de las universidades del Consejo de Rectores:

\begin{tabular}{llccc}
\hline Lugar & UNIVERSIDAD & $\begin{array}{c}\text { Puntaje PSU } \\
\text { promedio }\end{array}$ & $\begin{array}{c}\text { Diferencia con } \\
\text { postulación }\end{array}$ & $\begin{array}{c}\text { Cantidad de } \\
\text { estudiantes }\end{array}$ \\
\hline 1 & Pontificia Univ. Católica de Chile & 694,2 & 41,9 & 2.394 \\
2 & De Chile & 686,8 & 43,3 & 2.779 \\
3 & Téc. Federico Sta. María & 616,7 & 31 & 1.682 \\
4 & De Santiago de Chile & 614,7 & 32,7 & 1.956 \\
5 & Pontifica Univ. Católica de Valparaíso & 608,3 & 42,9 & 1.759 \\
6 & De Concepción & 606,1 & 40 & 2.988 \\
7 & De La Frontera & 594,8 & 45,3 & 890 \\
8 & Metropolitana de Ciencias de la Educ. & 593,5 & 45,1 & 408
\end{tabular}




\begin{tabular}{rlrrr}
9 & De Talca & 590,1 & 24 & 786 \\
10 & Tecnológica Metropolitana & 588,9 & 26,6 & 767 \\
11 & De Valparaíso & 583,4 & 28,5 & 1.855 \\
12 & Austral & 581,6 & 19,3 & 1.241 \\
13 & Católica del Norte & 573,4 & 22,7 & 1.118 \\
14 & Católica del Maule & 569,1 & 30,6 & 476 \\
15 & De La Serena & 553,9 & 33,3 & 987 \\
16 & Del Bío-Bío & 551,6 & 19,3 & 1.129 \\
17 & Católica de la Santísima Concepción & 551,5 & 10,5 & 677 \\
18 & De Playa Ancha & 550,0 & 742 \\
19 & De Antofagasta & 547,9 & 18,2 & 710 \\
20 & Magallanes & 537,8 & 19,7 & 378 \\
21 & Católica de Temuco & 534,6 & 16,5 & 826 \\
22 & De Tarapacá & 532,1 & 18,7 & 852 \\
23 & Arturo Prat & 526,2 & 21,2 & 643 \\
24 & De Atacama & 525,6 & 19,6 & 322 \\
25 & De Los Lagos & 524,4 & 19,5 & 541 \\
\hline
\end{tabular}

A su vez, es posible determinar que la distancia entre la universidad con mayor puntaje promedio (PUC) y la de menor puntaje (Universidad de Los Lagos) es de 169,8 puntos; lo anterior indica que los estudiantes discriminan al momento de postular, concentrándose los mejores puntajes en algunas universidades (ubicadas en las regiones Metropolitana, VIII y V). Dicho de otra manera, opera un fenómeno de segregación en algunas universidades, ya que éstas incorporan estudiantes de mayores puntajes. Este fenómeno se puede observar también al contrastar la diferencia de puntajes PSU entre el momento de postulación y el de matrícula (situación que se consigna en la penúltima columna de la tabla), ya que este hecho daría cuenta de un proceso de selección más exigente (en la medida en que quienes postulan con ponderaciones bajas no resultan ser, finalmente, alumnos de una determinada casa de estudios). Entonces, como tendencia, las instituciones que alcanzan un mayor promedio PSU son las mismas que presentan la mayor diferencia entre la postulación y matrícula (salvo la Universidad de La Serena). De este modo, es posible detectar diferencias por sobre los 40 puntos en la PUC, UCH, PUCV, UMCE y la Universidad de La Frontera (UFRO), mientras que en las universidades de bajo promedio la diferencia disminuye a menos de 20 puntos (ver parte inferior de la tabla precedente). 


\section{III.4. Matrícula en las universidades diferenciada por sexo del estudiante}

Esta es una variable que permite interrogar respecto de si existen universidades (y carreras) que tiendan a ser preferentemente "femeninas" o "masculinas". Con esta finalidad, se ordenaron las universidades en función de la cantidad y porcentaje de mujeres y hombres matriculados en ellas. En la tabla siguiente se presenta dicha información, iniciándose la tabla con la universidad que presenta mayor porcentaje de mujeres (UMCE, con un 65,2\%, seguida por la UCM) hasta la universidad donde existe el más bajo porcentaje de ellas (que, en este caso, corresponde a la UTFSM, con un $24,3 \%$; quedando esta casa de estudios con el mayor porcentaje de hombres: $75,7 \%$ ).

En la tabla siguiente se presentan la frecuencia y porcentajes de matriculados en cada universidad según su sexo:

\begin{tabular}{|c|c|c|c|c|c|c|}
\hline \multirow[b]{2}{*}{ Lugar } & \multirow[b]{2}{*}{ UNIVERSIDAD } & \multicolumn{2}{|c|}{ Femenino } & \multicolumn{2}{|c|}{ Masculino } & \multirow[b]{2}{*}{ Total } \\
\hline & & Frecuencia & Porcentaje & Frecuencia & Porcentaje & \\
\hline 1 & $\begin{array}{l}\text { Metropolitana de Ciencias } \\
\text { de la Educ. }\end{array}$ & 266 & $65,2 \%$ & 142 & $34,8 \%$ & 408 \\
\hline 2 & Católica del Maule & 304 & $63,9 \%$ & 172 & $36,1 \%$ & 476 \\
\hline 3 & Católica de Temuco & 499 & $60,4 \%$ & 327 & $39,6 \%$ & 826 \\
\hline 4 & Católica de la Sant. Concepción & 399 & $58,9 \%$ & 278 & $41,1 \%$ & 677 \\
\hline 5 & De Playa Ancha & 429 & $57,8 \%$ & 313 & $42,2 \%$ & 742 \\
\hline 6 & De Los Lagos & 309 & $57,1 \%$ & 232 & $42,9 \%$ & 541 \\
\hline 7 & Arturo Prat & 365 & $56,8 \%$ & 278 & $43,2 \%$ & 643 \\
\hline 8 & De Valparaíso & 1.024 & $55,2 \%$ & 831 & $44,8 \%$ & 1.855 \\
\hline 9 & De Tarapacá & 470 & $55,2 \%$ & 382 & $44,8 \%$ & 852 \\
\hline 10 & De Antofagasta & 384 & $54,1 \%$ & 326 & $45,9 \%$ & 710 \\
\hline 11 & De Magallanes & 198 & $52,4 \%$ & 180 & $47,6 \%$ & 378 \\
\hline 12 & De La Serena & 494 & $50,1 \%$ & 493 & $49,9 \%$ & 987 \\
\hline 13 & De Concepción & 1.472 & $49,3 \%$ & 1.516 & $50,7 \%$ & 2.988 \\
\hline 14 & $\begin{array}{l}\text { Pontificia Univ. Católica } \\
\text { de Chile }\end{array}$ & 1.172 & $49,0 \%$ & 1.222 & $51,0 \%$ & 2.394 \\
\hline 15 & $\begin{array}{l}\text { Pontifica Univ. Católica } \\
\text { de Valparaíso }\end{array}$ & 840 & $47,8 \%$ & 919 & $52,2 \%$ & 1.759 \\
\hline 16 & Austral & 590 & $47,5 \%$ & 651 & $52,5 \%$ & 1.241 \\
\hline 17 & De Talca & 371 & $47,2 \%$ & 415 & $52,8 \%$ & 786 \\
\hline 18 & Del Norte & 524 & $46,9 \%$ & 594 & $53,1 \%$ & 1.118 \\
\hline 19 & De Chile & 1.289 & $46,4 \%$ & 1.490 & $53,6 \%$ & 2.779 \\
\hline 20 & De Atacama & 145 & $45,0 \%$ & 177 & $55,0 \%$ & 322 \\
\hline
\end{tabular}




\begin{tabular}{|c|c|c|c|c|c|}
\hline De La Frontera & 398 & $44,7 \%$ & 492 & $55,3 \%$ & 890 \\
\hline De Santiago & 861 & $44,0 \%$ & 1.095 & $56,0 \%$ & 1.956 \\
\hline Del Bío-Bío & 468 & $41,5 \%$ & 661 & $58,5 \%$ & 1.129 \\
\hline Tecnológica Metropolitana & 303 & $39,5 \%$ & 464 & $60,5 \%$ & 767 \\
\hline Téc. Federico Sta. María & 408 & $24,3 \%$ & 1.274 & $75,7 \%$ & 1.682 \\
\hline Total & 13.982 & $48,4 \%$ & 14.924 & $51,6 \%$ & 28.906 \\
\hline
\end{tabular}

Al considerar el total de estudiantes matriculados, existe un cierto equilibrio en cuanto a participación en la educación superior por sexo (ya que el $48,4 \%$ de los estudiantes corresponde a mujeres y el $51,6 \%$ a hombres). No obstante, si se consideran los datos provenientes de los procesos de rendición y postulación a la PSU, es posible afirmar que en el proceso de matrícula existiría una subrepresentación de la población femenina. Ello porque una mayor cantidad de mujeres rendía PSU (de los 122.014 inscritos, 64.349 eran mujeres) y registraba postulación a las universidades en primera preferencia (26.266 mujeres v/s 25.445 hombres).

Por otra parte, la distribución por sexo para cada universidad resulta una variable interesante en la medida en que muestra que algunos establecimientos de educación superior son elegidos con un patrón de género. Así, existen universidades (UMCE, UCM y Católica de Temuco (UCT)) con más del 60\% total de matrícula femenina; mientras que dos establecimientos (UTFSM y Universidad Tecnológica Metropolitana, UTEM) tienen niveles de matrícula masculina superior al 60\%. Lo anterior, puede relacionarse con el tipo de carreras que ofrecen ambos grupos de universidades: las tres universidades con mayoría de matrícula femenina ofrecen una importante cantidad de carreras de pedagogía; a su vez, la UTEM y la UTFSM ofrecen carreras del área de la ingeniería y de carácter técnico.

\section{III.5. Matrícula por rama educacional}

Los estudiantes aparecen clasificados según el ingreso familiar reportado al momento de rendir la PSU. Las respuestas entregadas fueron segmentadas en seis grupos de ingreso, de acuerdo con las categorías determinadas por el DEMRE: 
- G1: Ingreso bruto familiar inferior a 278.000 pesos

- G2: Ingreso bruto familiar fluctúa entre 278.001 a 834.000 pesos.

- G3: Ingreso bruto familiar fluctúa entre 834.001 a 1.400 .000 pesos.

- G4: Ingreso bruto familiar fluctúa entre 1.400 .001 a 1.950 .000 pesos

- G5: Ingreso bruto familiar fluctúa entre 1.950 .000 a 2.500 .000 pesos

- G6: Ingreso bruto familiar superior a 2.500 .000 pesos.

En el gráfico que se presenta a continuación se puede apreciar la distribución socioeconómica de los estudiantes que se matriculan en las universidades del Consejo de Rectores.

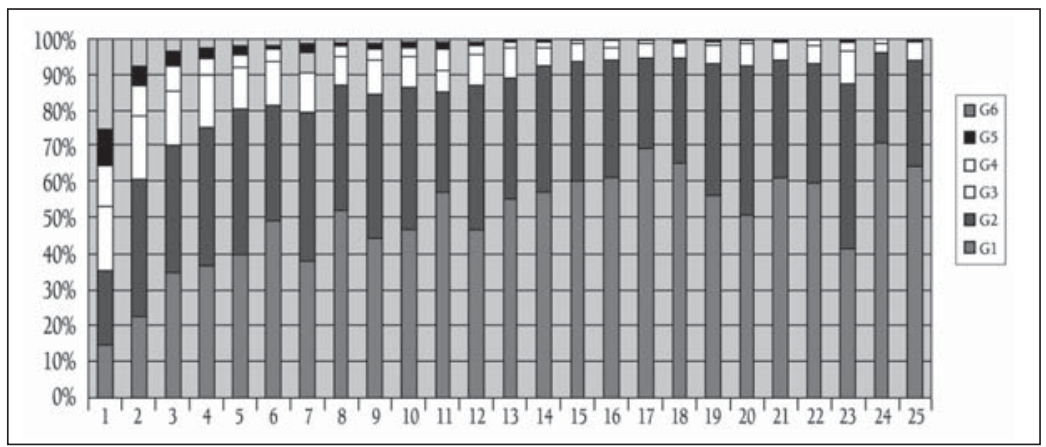

La información más detallada en cuanto a los códigos de cada universidad y los porcentajes exactos en que cada grupo socioeconómico queda representado en esas instituciones se observa en la siguiente tabla que presenta el porcentaje de matriculados en cada universidad, según el ingreso familiar reportado:

\begin{tabular}{|c|c|c|c|c|c|c|c|c|c|c|c|c|}
\hline Lugar & UNIVERSIDAD & G l & $\%$ & $G_{2}$ & $\%$ & G 3 & $\%$ & B 4 & $\%$ G 5 & $\%$ & G 6 & $\%$ \\
\hline \multirow[t]{2}{*}{1} & Pontificia Univ. & & & & & & & & & & & \\
\hline & Católica de Chile & 354 & $14,8 \%$ & 495 & $20,7 \%$ & 418 & $17,5 \%$ & 283 & $11,8 \% 236$ & $9,9 \%$ & 608 & $25,4 \%$ \\
\hline 2 & De Chile & 638 & $23,0 \%$ & 1.048 & $37,7 \%$ & 485 & $17,5 \%$ & 239 & $8,6 \% 161$ & $5,8 \%$ & 208 & $7,5 \%$ \\
\hline 3 & $\begin{array}{l}\text { Téc. Federico } \\
\text { Sta. María }\end{array}$ & 589 & $35,0 \%$ & 596 & $35,4 \%$ & 254 & $15,1 \%$ & 116 & $6,9 \% \quad 64$ & $3,8 \%$ & 63 & $3,7 \%$ \\
\hline 4 & $\begin{array}{l}\text { Pontificia Univ. } \\
\text { Católica de Valparaíso }\end{array}$ & 646 & $36,7 \%$ & 682 & $38,8 \%$ & 254 & $14,4 \%$ & 80 & $4,5 \% \quad 51$ & $2,9 \%$ & 46 & $2,6 \%$ \\
\hline 5 & De Antofagasta & 283 & $39,9 \%$ & 286 & $40,3 \%$ & 82 & $11,5 \%$ & 26 & $3,7 \% \quad 19$ & $2,7 \%$ & 14 & $2,0 \%$ \\
\hline 6 & De Concepción & 1.462 & $48,9 \%$ & 967 & $32,4 \%$ & 359 & $12,0 \%$ & 106 & $3,5 \% \quad 41$ & $1,4 \%$ & 53 & $1,8 \%$ \\
\hline 7 & Católica del Norte & 426 & $38,1 \%$ & 461 & $41,2 \%$ & 125 & $11,2 \%$ & 60 & $5,4 \% \quad 28$ & $2,5 \%$ & 18 & $1,6 \%$ \\
\hline
\end{tabular}




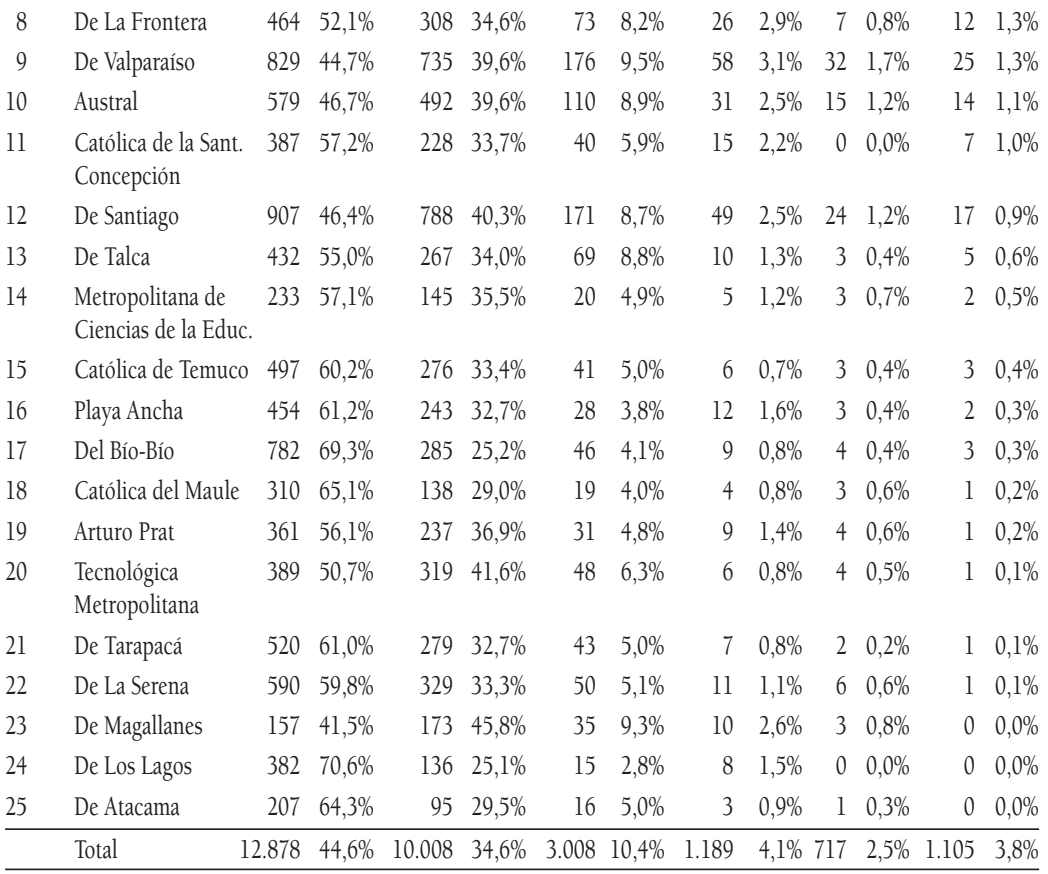

Como se puede observar, las universidades incorporan estudiantes con perfiles socioeconómicos disímiles, ya que la única universidad que registra una población de ingreso que incluye cada uno de los diferentes niveles de ingresos de manera relativamente equitativa es, a su vez, la que ocupa la primera posición en los gráficos y tablas recién presentados (PUC). A su vez, dicha institución incorpora la más importante representación de los tres grupos de mayores ingresos y que cuenta en sus aulas con un $25 \%$ de estudiantes con ingresos superiores a $\$ 2.500 .000$ (grupo G6 en la tabla anterior). Asimismo, la propia PUC es la universidad que posee la más baja cantidad de estudiantes cuyos ingresos familiares son menores a \$278.000. En efecto, en esa universidad casi la mitad de los matriculados reporta ingresos superiores a 1.400 .000 pesos mensuales, situación especialmente diferente en relación con las restantes instituciones. 
Por otra parte, las universidades que se ubican hacia la derecha del gráfico poseen una proporción significativamente mayor de estudiantes con ingresos familiares inferiores a 834.000 pesos (grupos G1 y G2, respectivamente). En términos concretos, las universidades de Los Lagos, del Bío- Bío y del Maule (representadas en el gráfico con los números 24,17 y 18 , respectivamente) poseen una matrícula de alumnos pobres superior al $60 \%$ de su total. Al respecto, hay que resaltar que el $80 \%$ de los estudiantes con ingresos inferiores a $\$ 840.000$ se reparte en un total de 21 universidades.

A partir de toda la información ya señalada, es posible concluir que las universidades congregan a alumnos con ingresos familiares diferentes, ya que los estudiantes con altos ingresos económicos postulan de preferencia a las instituciones que incorporan altos puntajes promedio en PSU (ver tercer acápite).

\section{III.6. Dependencia por universidades}

De manera similar a lo que ocurre con los ingresos familiares autorreportados, la composición de las universidades, según la dependencia de los establecimientos educacionales, exhibe un patrón bastante marcado que facilita la distinción entre las instituciones. De este modo, determinadas universidades convocan jóvenes principalmente de colegios particulares pagados, mientras que en las restantes instituciones el porcentaje de dichos estudiantes disminuye notoriamente, en beneficio de los establecimientos subvencionados. Con el objeto de reflejar dicha situación, se confeccionó el siguiente gráfico, donde las universidades se ordenan en función de la cantidad de alumnos matriculados provenientes de establecimientos particulares pagados. 


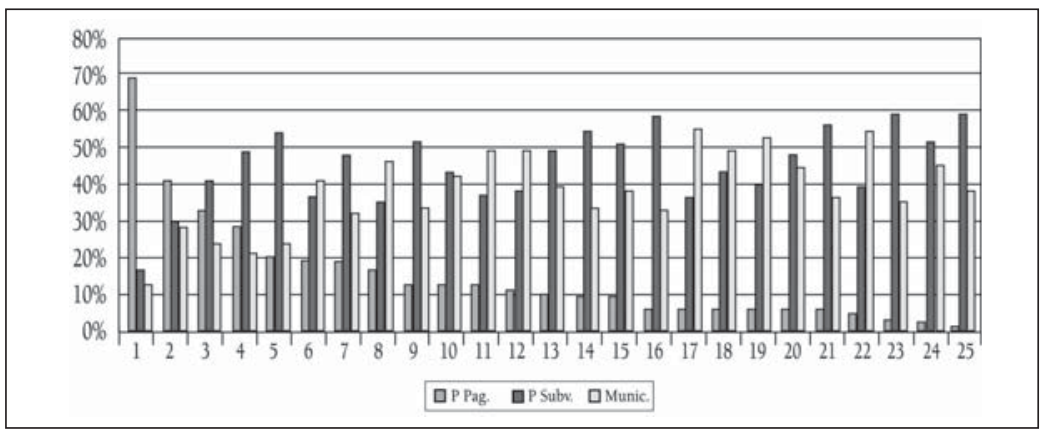

Para facilitar la comprensión del gráfico, se adjunta a continuación la tabla con el porcentaje de casos matriculados en función de la dependencia del colegio de egreso. Esta tabla, junto con fundamentar el gráfico y entregar el código que identifica a cada institución, detalla la cantidad de estudiantes que se matriculan en cada institución en función de la dependencia educacional.

\begin{tabular}{|c|c|c|c|c|c|c|c|c|}
\hline \multirow[b]{2}{*}{ Lugar } & \multirow[b]{2}{*}{ UNIVERSIDAD } & \multicolumn{2}{|c|}{$\begin{array}{l}\text { Particular } \\
\text { Pagado }\end{array}$} & \multicolumn{2}{|c|}{$\begin{array}{c}\text { Particular } \\
\text { Subvencionado }\end{array}$} & \multicolumn{2}{|c|}{ Municipal } & \multirow[b]{2}{*}{ Total } \\
\hline & & Casos & $\%$ & Casos & $\%$ & Casos & $\%$ & \\
\hline 1 & $\begin{array}{l}\text { Pontificia Univ. } \\
\text { Católica de Chile }\end{array}$ & 1.680 & $70,2 \%$ & 408 & $17,0 \%$ & 306 & $12,8 \%$ & 2.394 \\
\hline 2 & De Chile & 1.149 & $41,3 \%$ & 817 & $29,4 \%$ & 813 & $29,3 \%$ & 2.779 \\
\hline 3 & Téc. Federico Sta. María & 562 & $33,4 \%$ & 703 & $41,8 \%$ & 417 & $24,8 \%$ & 1.682 \\
\hline 4 & $\begin{array}{l}\text { Pontifica Univ. } \\
\text { Católica de Valparaíso }\end{array}$ & 506 & $28,8 \%$ & 877 & $49,9 \%$ & 376 & $21,4 \%$ & 1.759 \\
\hline 5 & De Valparaíso & 390 & $21,0 \%$ & 1.015 & $54,7 \%$ & 450 & $24,3 \%$ & 1.855 \\
\hline 6 & De Concepción & 605 & $20,2 \%$ & 1.118 & $37,4 \%$ & 1.265 & $42,3 \%$ & 2.988 \\
\hline 7 & Católica del Norte & 214 & $19,1 \%$ & 543 & $48,6 \%$ & 361 & $32,3 \%$ & 1.118 \\
\hline 8 & De Antofagasta & 123 & $17,3 \%$ & 255 & $35,9 \%$ & 332 & $46,8 \%$ & 710 \\
\hline 9 & De Santiago & 257 & $13,1 \%$ & 1.030 & $52,7 \%$ & 669 & $34,2 \%$ & 1.956 \\
\hline 10 & Austral & 162 & $13,1 \%$ & 543 & $43,8 \%$ & 536 & $43,2 \%$ & 1.241 \\
\hline 11 & $\begin{array}{l}\text { Católica de la Santísima } \\
\text { Concepción }\end{array}$ & 88 & $13,0 \%$ & 253 & $37,4 \%$ & 336 & $49,6 \%$ & 677 \\
\hline 12 & De Talca & 94 & $12,0 \%$ & 303 & $38,5 \%$ & 389 & $49,5 \%$ & 786 \\
\hline 13 & De Magallanes & 41 & $10,8 \%$ & 187 & $49,5 \%$ & 150 & $39,7 \%$ & 378 \\
\hline 14 & De La Frontera & 94 & $10,6 \%$ & 491 & $55,2 \%$ & 305 & $34,3 \%$ & 890 \\
\hline 15 & $\begin{array}{l}\text { Metropolitana de } \\
\text { Ciencias de la Educ. }\end{array}$ & 41 & $10,0 \%$ & 211 & $51,7 \%$ & 156 & $38,2 \%$ & 408 \\
\hline 16 & De Playa Ancha & 53 & $7,1 \%$ & 441 & $59,4 \%$ & 248 & $33,4 \%$ & 742 \\
\hline 17 & Católica del Maule & 32 & $6,7 \%$ & 178 & $37,4 \%$ & 266 & $55,9 \%$ & 476 \\
\hline 18 & De Tarapacá & 52 & $6,1 \%$ & 377 & $44,2 \%$ & 423 & $49,6 \%$ & 852 \\
\hline 19 & Del Bío-Bío & 67 & $5,9 \%$ & 464 & $41,1 \%$ & 598 & $53,0 \%$ & 1.129 \\
\hline
\end{tabular}




\begin{tabular}{llrrrrrrr}
20 & Católica de Temuco & 49 & $5,9 \%$ & 404 & $48,9 \%$ & 373 & $45,2 \%$ & 826 \\
21 & Tecnológica Metropolitana & 45 & $5,9 \%$ & 435 & $56,7 \%$ & 287 & $37,4 \%$ & 767 \\
22 & De Los Lagos & 24 & $4,4 \%$ & 215 & $39,7 \%$ & 302 & $55,8 \%$ & 541 \\
23 & De La Serena & 34 & $3,4 \%$ & 599 & $60,7 \%$ & 354 & $35,9 \%$ & 987 \\
24 & De Atacama & 7 & $2,2 \%$ & 168 & $52,2 \%$ & 147 & $45,7 \%$ & 322 \\
25 & Arturo Prat & 7 & $1,1 \%$ & 387 & $60,2 \%$ & 249 & $38,7 \%$ & 643 \\
\hline & Total & 6.376 & & 12.422 & & 10.108 & & 28.906 \\
\hline
\end{tabular}

Como ya se mencionó en el primer capítulo, las universidades reciben mayoritariamente estudiantes de colegios particulares subvencionados y municipales. Concretamente, 12.422 alumnos matriculados provenían de establecimientos subvencionados, lo que representa el $43 \%$ del total de casos (recordando que el total equivale a 28.906 jóvenes). Esta tendencia encuentra su excepción en la PUC y en la UCH (números 1 y 2 en el gráfico), donde la proporción de estudiantes de colegios particulares pagados es mayor que la de particulares subvencionados y municipales. Aún más, si se suma la cantidad de estudiantes provenientes de colegios municipales y particulares subvencionados en todas las universidades, el resultado es una cantidad superior al alumnado egresado de colegios particulares, salvo en la PUC (donde sólo un 29,8\% no proviene de este tipo de colegios). Como se puede observar en el gráfico recién presentado (de izquierda a derecha), las demás casas de estudio integran progresivamente, y de manera proporcional, estudiantes que provienen de establecimientos subvencionados. Asimismo, las únicas universidades donde más de la mitad de sus matriculados provienen de colegios municipales corresponden a la UCM $(55,9 \%)$, de Los Lagos (55,8\%) y del Bío-Bío (53\%).

Por último, es importante señalar que las universidades de La Serena, de Atacama y Arturo Prat (todas ubicadas en la zona norte del país) integran una proporción minoritaria de estudiantes de colegios particulares pagados (proporción inferior al 5\%).

\section{III.7. Matrícula por rama educacional}

Para analizar la distribución de matriculados de cada universidad en función de la rama educacional del colegio de egreso de los alumnos, 
se debe considerar, en primer lugar, que los estudiantes de educación media que estudian en colegios técnico-profesionales (TP) no estarían totalmente orientados a continuar su formación en la universidad. Esta premisa se cumple de manera relativa y desigual entre las diferentes universidades. De esta manera, hay algunas instituciones que prácticamente completan su matrícula en forma exclusiva con estudiantes provenientes de los establecimientos científicohumanistas (en la PUC y en la UCH más del 98\% proviene de este tipo de establecimientos), lo cual se observa en la siguiente tabla de distribución de la matrícula según rama educacional:

\begin{tabular}{|c|c|c|c|c|c|c|}
\hline & Universidad & $\begin{array}{l}\text { Científico } \\
\text { humanista }\end{array}$ & $\%$ & $\begin{array}{c}\text { Técnico } \\
\text { profesional }\end{array}$ & $\%$ & Total \\
\hline 1 & Pontificia Univ. Católica de Chile & 2.379 & $99,4 \%$ & 15 & $0,6 \%$ & 2.394 \\
\hline 2 & De Chile & 2.746 & $98,8 \%$ & 33 & $1,2 \%$ & 2.779 \\
\hline 3 & Pontifica Univ. Católica de Valparaíso & 1.641 & $93,3 \%$ & 118 & $6,7 \%$ & 1.759 \\
\hline 4 & Austral & 1.151 & $92,7 \%$ & 90 & $7,3 \%$ & 1.241 \\
\hline 5 & De Valparaíso & 1.719 & $92,7 \%$ & 136 & $7,3 \%$ & 1.855 \\
\hline 6 & De Playa Ancha & 685 & $92,3 \%$ & 57 & $7,7 \%$ & 742 \\
\hline 7 & Católica de Temuco & 762 & $92,3 \%$ & 64 & $7,7 \%$ & 826 \\
\hline 8 & De La Frontera & 811 & $91,1 \%$ & 79 & $8,9 \%$ & 890 \\
\hline 9 & Téc. Federico Sta. María & 1.527 & $90,8 \%$ & 155 & $9,2 \%$ & 1.682 \\
\hline 10 & De Santiago & 1.771 & $90,5 \%$ & 185 & $9,5 \%$ & 1.956 \\
\hline 11 & De La Serena & 891 & $90,3 \%$ & 96 & $9,7 \%$ & 987 \\
\hline 12 & De Concepción & 2.696 & $90,2 \%$ & 292 & $9,8 \%$ & 2.988 \\
\hline 13 & Católica del Maule & 425 & $89,3 \%$ & 51 & $10,7 \%$ & 476 \\
\hline 14 & Católica del Norte & 998 & $89,3 \%$ & 120 & $10,7 \%$ & 1.118 \\
\hline 15 & De Tarapacá & 759 & $89,1 \%$ & 93 & $10,9 \%$ & 852 \\
\hline 16 & Metropolitana de Ciencias de la Educ. & 362 & $88,7 \%$ & 46 & $11,3 \%$ & 408 \\
\hline 17 & Católica de la Santísima Concepción & 596 & $88,0 \%$ & 81 & $12,0 \%$ & 677 \\
\hline 18 & De Antofagasta & 621 & $87,5 \%$ & 89 & $12,5 \%$ & 710 \\
\hline 19 & De Talca & 682 & $86,8 \%$ & 104 & $13,2 \%$ & 786 \\
\hline 20 & De Magallanes & 327 & $86,5 \%$ & 51 & $13,5 \%$ & 378 \\
\hline 21 & De Los Lagos & 455 & $84,1 \%$ & 86 & $15,9 \%$ & 541 \\
\hline 22 & Tecnológica Metropolitana & 636 & $82,9 \%$ & 131 & $17,1 \%$ & 767 \\
\hline 23 & Arturo Prat & 500 & $77,8 \%$ & 143 & $22,2 \%$ & 643 \\
\hline 24 & Del Bío-Bío & 828 & $73,3 \%$ & 301 & $26,7 \%$ & 1.129 \\
\hline \multirow[t]{2}{*}{25} & De Atacama & 144 & $44,7 \%$ & 178 & $55,3 \%$ & 322 \\
\hline & Total & 26.112 & $90,3 \%$ & 2794 & $9,7 \%$ & 28.906 \\
\hline
\end{tabular}

La mayoría de las restantes universidades presentan una proporción de estudiantes similar a la encontrada en el total de matriculados (90\% de científico-humanistas y $10 \%$ de técnico- 
profesional, aproximadamente), aspecto ya reseñado en el primer punto de este capítulo. No obstante, existen tres universidades en que más del 20\% de su alumnado proviene de establecimientos TP: Arturo Prat, del Bío-Bío y de Atacama (ubicadas en la parte baja de la tabla); incluso la última incorpora más matrícula proveniente de TP, siendo la única institución universitaria en que se presenta esta proporción.

Para entender esta situación se verificó el tipo de carreras impartidas por estas instituciones. Se determinó que, tanto la Universidad de Atacama como la Universidad Arturo Prat son instituciones en que cerca de la mitad de las carreras ofrecidas poseen un carácter técnico ${ }^{\text {iv }}$, lo que no ocurre en la Universidad del Bío-Bío.

\section{III.8. Distribución de matrícula por regiones}

Para analizar la composición de los estudiantes que se matriculan en una institución en función de su región de procedencia, se debe tener en cuenta si las casas de estudios escogidas poseen o no sedes en otras regiones. Este evento permite entender por qué algunas universidades tienen un alto volumen de alumnos provenientes de regiones distantes al lugar donde ofrecen determinadas carreras. A su vez, tampoco se debe olvidar el particular caso de la VI Región, la que, por no contar con una universidad del Consejo de Rectores en su territorio, experimenta una emigración de sus estudiantes. Una situación similar se vive en la XI Región, la cual tampoco cuenta con universidades instaladas en su territorio. Sin embargo, esta situación se subsana con la existencia de una sede regional de la Universidad Austral. En todo caso, el número de matriculados que aporta la XI Región es menor al $0,5 \%$ del total país, por lo que no afecta el análisis global.

La tabla que a continuación se presenta puede ser leída horizontalmente (por filas), lo que permite observar el origen regional de los estudiantes de cada una de las universidades, y verticalmente (por columnas), donde el énfasis estará puesto en el lugar de matrícula de los alumnos que vive en cada región.

iv La Universidad de Atacama imparte 27 carreras, de las cuales 13 (48\%) son de tipo técnico. Asimismo, la Universidad Arturo Prat posee 26 carreras técnicas de un total de 56 (equivalen al $46 \%$ del total). 


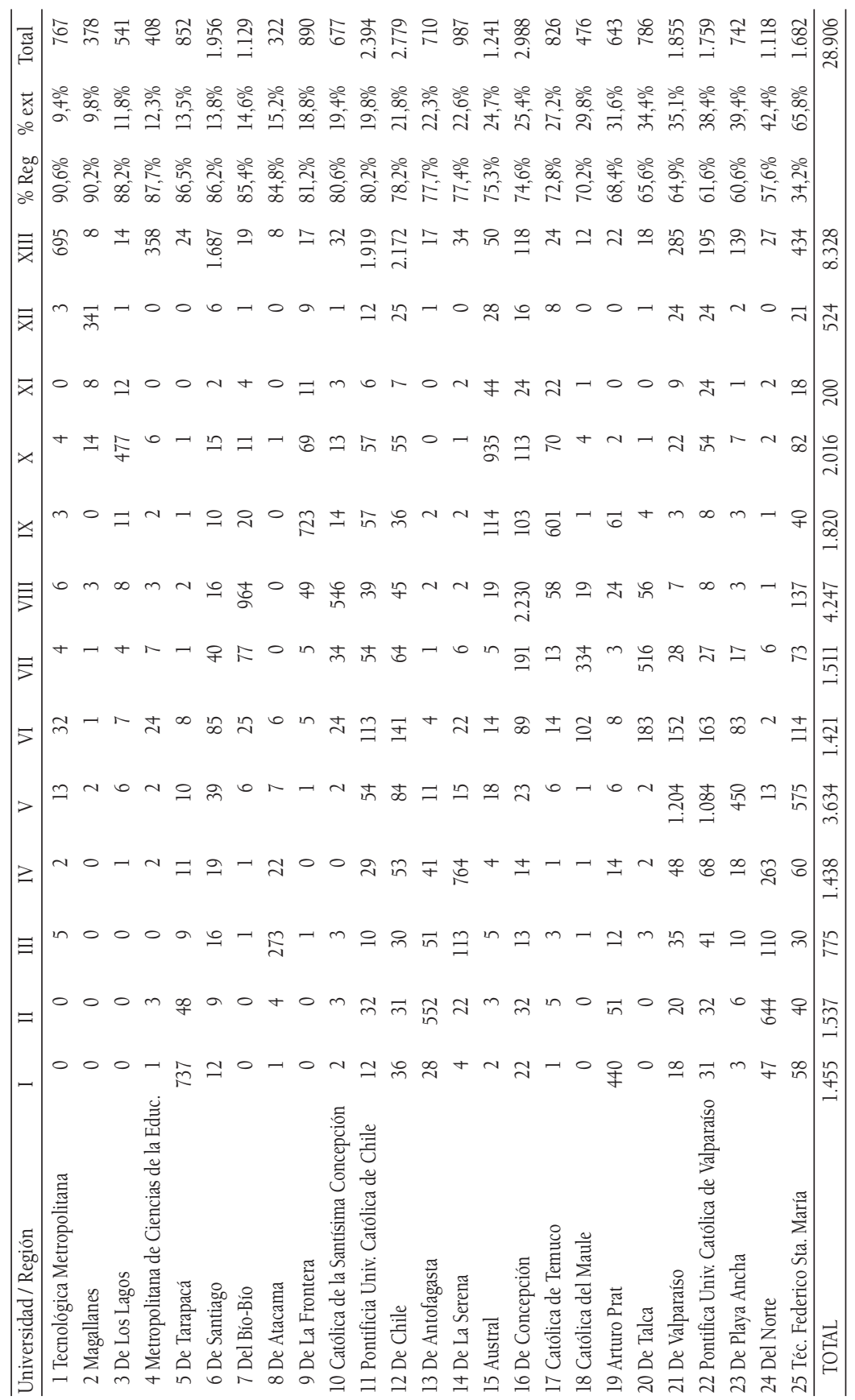


Antes que nada, es preciso señalar que las universidades incorporan mayoritariamente estudiantes que provienen de la misma región donde se instalan. Para facilitar este análisis, se construyeron en el extremo derecho de la tabla dos columnas que presentan tanto el porcentaje de alumnos que proviene de la misma región (\% Reg) como el que proviene de una región externa (\% ext). Al respecto, se debe señalar que tanto la UTFSM como la UCN poseen sedes regionales, mientras que la PUCV y las universidades de Valparaíso y de Playa Ancha reciben un numeroso aporte de estudiantes provenientes de la Región Metropolitana (ubicada a tan sólo 120 kilómetros de la ciudad de Valparaíso) y de la VI Región (donde no existen universidades).

Hay un conjunto de universidades que tienen sede en una región y que, pese a ello, incorporan un importante contingente de estudiantes de otras regiones (entre el 19\% y el 30\% del total de su matrícula), situación propia de las universidades en la parte central de la tabla (lugares 10 a 18). Es posible pensar que este grupo deba la presencia de estudiantes de otras regiones a su prestigio y/o a la cercanía con éstas.

Finalmente, al desarrollar un análisis para cada región, se determina que sólo las regiones V, VI y Metropolitana aportan estudiantes a cada una de las universidades reunidas en el Consejo de Rectores.

Debido a que no poseen universidades en su lugar de residencia, los estudiantes de la VI Región deben emigrar principalmente a instituciones con sedes en las regiones $\mathrm{V}$, Metropolitana y VII, mientras que la V y la Región Metropolitana retienen a la mayoría de sus estudiantes dentro de sus límites.

\section{III.9. Nivel educacional del padre}

La información relativa al nivel educacional de los padres de los estudiantes matriculados se presenta ordenada en función del porcentaje de alumnos cuyo padre alcanza educación universitaria 
(sea esta completa o no). De este modo, se determina que del total de estudiantes que se matricula en la Pontificia Universidad Católica, en el $70,3 \%$ de los casos su padre posee niveles de educación universitario. Por esta razón, en la tabla que se presenta a continuación la PUC ostenta el primer lugar. Además de dicha institución, sólo en la Universidad de Chile se detecta que más de la mitad de los padres de los estudiantes matriculados poseen educación universitaria. Para realizar este análisis se dejaron de lado todos aquellos sujetos que no aportaron información al respecto.

\begin{tabular}{llcccc}
\hline & UNIVERSIDAD & $\begin{array}{c}\text { \% Educ. básica } \\
\text { o menos }\end{array}$ & \% Educ. media & $\begin{array}{c}\text { \% Técnico- } \\
\text { profesional }\end{array}$ & \% Universit. \\
\hline 1 & Pontificia Univ. Católica de Chile & 5,9 & 16,1 & 7,7 & 70,3 \\
2 & De Chile & 6,9 & 25,5 & 10,9 & 56,7 \\
3 & Téc. Federico Sta. María & 10,8 & 34,4 & 10,1 & 44,7 \\
4 & Pontifica Univ. Católica de Valparaíso & 13,9 & 36,2 & 10,9 & 39,0 \\
5 & De Concepción & 15,0 & 39,8 & 7,1 & 38,1 \\
6 & De Antofagasta & 12,7 & 43,5 & 7,2 & 36,6 \\
7 & Católica del Norte & 11,1 & 42,7 & 9,7 & 36,5 \\
8 & De Valparaíso & 12,3 & 42,8 & 11,5 & 33,4 \\
9 & Austral & 16,7 & 42,4 & 8,4 & 32,5 \\
10 & De La Frontera & 16,1 & 44,2 & 7,1 & 32,5 \\
11 & De Santiago & 13,0 & 44,6 & 11,4 & 30,9 \\
12 & Católica de la Santísima Concepción & 15,3 & 46,3 & 8,7 & 29,7 \\
13 & De Talca & 20,7 & 42,0 & 9,9 & 27,3 \\
14 & Arturo Prat & 13,5 & 52,5 & 8,0 & 26,0 \\
15 & De Playa Ancha & 16,8 & 49,3 & 8,3 & 25,5 \\
16 & De Tarapacá & 14,6 & 52,9 & 7,3 & 25,2 \\
17 & De La Serena & 16,7 & 49,5 & 8,7 & 25,0 \\
18 & Metropolitana de Ciencias de la Educ. & 17,3 & 47,3 & 10,7 & 24,7 \\
19 & Tecnológica Metropolitana & 14,0 & 49,6 & 11,9 & 24,6 \\
20 & Católica de Temuco & 17,3 & 51,3 & 7,4 & 23,9 \\
21 & De Atacama & 21,9 & 47,9 & 9,0 & 21,2 \\
22 & De Magallanes & 22,1 & 49,7 & 7,2 & 21,0 \\
23 & Del Bío-Bío & 50,2 & 4,6 & 20,9 \\
24 & Católica del Maule & 24,2 & 50,5 & 8,2 & 17,4 \\
25 & De Los Lagos & 23,9 & 52,8 & 5,2 & 16,5 \\
\hline
\end{tabular}

Es posible destacar que las cinco instituciones con más bajos porcentajes de alumnos con padres universitarios son las universidades de Atacama, Magallanes, del Bío-Bío, del Maule y de Los Lagos, todos centros educacionales no ubicados en la región 
capital del país. Asimismo, se debe mencionar que la misma Universidad de Los Lagos aglutina al mayor porcentaje de alumnos cuyos padres tienen nivel educacional más bajo (el 25\% posee educación básica o un nivel de instrucción menor). Finalmente, con excepción de la PUC y la UCH, en todas las restantes universidades lo más frecuente es encontrar padres cuyo grado educacional corresponde a enseñanza media (completa o no).

\section{III.10. Nivel educacional de la madre}

Al igual que con los padres, se determina que es la PUC la institución que alcanza el mayor porcentaje de alumnos cuyas madres poseen educación universitaria (alrededor del 60\%), pero esta vez es la única que supera el 50\%. Pese a ello, la UCH también alcanza la segunda mayoría (ya que el 45,1\% de las madres de los alumnos de dicha casa de estudios posee nivel universitario, completo o no), tal como se aprecia en la siguiente tabla de porcentaje de matriculados según el nivel educacional de la madre:

\begin{tabular}{|c|c|c|c|c|c|}
\hline \multicolumn{2}{|c|}{ UNIVERSIDAD } & $\begin{array}{l}\text { \% Educ. básica } \\
\text { o menos }\end{array}$ & \multirow{2}{*}{$\begin{array}{c}\text { \% Educ. media } \\
22,0\end{array}$} & \multirow{2}{*}{$\begin{array}{c}\text { \% Técnico- } \\
\text { profesional }\end{array}$} & \multirow{2}{*}{$\begin{array}{c}\% \text { Universit. } \\
60,4\end{array}$} \\
\hline 1 & Pontificia Univ. Católica de Chile & 5,0 & & & \\
\hline 2 & De Chile & 7,0 & 34,1 & 13,8 & 45,1 \\
\hline 3 & Téc. Federico Sta. María & 10,1 & 42,5 & 13,9 & 33,6 \\
\hline 4 & De Concepción & 14,1 & 45,3 & 9,3 & 31,4 \\
\hline 5 & Pontifica Univ. Católica de Valparaíso & so $\quad 10,8$ & 45,4 & 12,6 & 31,2 \\
\hline 6 & De La Frontera & 14,8 & 49,1 & 6,9 & 29,2 \\
\hline 7 & Austral & 15,9 & 47,6 & 10,0 & 26,5 \\
\hline 8 & De Talca & 16,1 & 48,4 & 9,4 & 26,1 \\
\hline 9 & De Valparaíso & 12,2 & 47,3 & 14,8 & 25,7 \\
\hline 10 & Católica del Norte & 10,0 & 52,6 & 11,9 & 25,5 \\
\hline 11 & De Antofagasta & 13,2 & 53,3 & 9,4 & 24,2 \\
\hline 12 & De Santiago & 13,6 & 51,3 & 12,6 & 22,4 \\
\hline 13 & Católica de Temuco & 17,3 & 51,6 & 9,4 & 21,7 \\
\hline 14 & Católica de la Santísima Concepción & 17,7 & 50,5 & 11,3 & 20,5 \\
\hline 15 & De Tarapacá & 17,0 & 56,0 & 7,0 & 19,9 \\
\hline 16 & Metropolitana de Ciencias de la Educ. & ac. 15,7 & 53,4 & 12,1 & 18,8 \\
\hline 17 & De La Serena & 15,5 & 55,5 & 11,4 & 17,6 \\
\hline 18 & Católica del Maule & 22,5 & 54,4 & 5,9 & 17,2 \\
\hline 19 & De Playa Ancha & 17,2 & 52,5 & 13,3 & 17,1 \\
\hline 20 & Tecnológica Metropolitana & 11,6 & 56,7 & 14,8 & 16,9 \\
\hline 21 & Arturo Prat & 13,8 & 59,8 & 9,8 & 16,7 \\
\hline
\end{tabular}




\begin{tabular}{|c|c|c|c|c|}
\hline 22 De Magallanes & 19,2 & 57,1 & 7,0 & 16,7 \\
\hline De Los Lagos & 26,7 & 53,8 & 3,8 & 15,6 \\
\hline Del Bío-Bío & 20,9 & 56,5 & 7,2 & 15,4 \\
\hline De Atacama & 18,8 & 59,2 & 7,6 & 14,3 \\
\hline
\end{tabular}

Se aprecia que la mayoría de las madres de estudiantes matriculados -con excepción de las dos universidades ya mencionadas- no alcanzan niveles educativos de tipo técnico o universitario. Por otra parte, la Universidad de Los Lagos es la que presenta la mayor cantidad de alumnos cuyas madres sólo poseen hasta educación básica (incluyéndose en esta categoría aquellas que no tienen escolaridad). No obstante, esta institución no es un fiel reflejo del nivel de las madres, ya que el porcentaje de casos que posee nivel básico resulta inferior al porcentaje de padres que sólo poseen hasta esa categoría de estudios.

Nuevamente, se debe destacar que los casos "sin información" fueron descartados de forma previa a los análisis estadísticos de los datos (es decir, fueron excluidos del proceso).

\section{III.11. Ocupación principal del padre}

En primer lugar, debido a la extensa categorización existente respecto a la ocupación principal del padre (la que incluía doce posibilidades), y para lograr un análisis de datos adecuado, fue necesario reducirla a tres categorías. Así, en la primera categoría se incluyó a los gerentes, administrativos, grandes empresarios y profesionales con cinco años o más de estudios universitarios. En la segunda categoría (denominada ahora "empleados") se agrupó a empleados especializados, de oficina, pequeños empresarios y miembros de las fuerzas armadas no oficiales. La tercera categoría, llamada "oficio/obrero", incluye las restantes posibilidades.

Hecha esta recategorización, es posible observar en la tabla que se presenta a continuación cómo los hijos de directivos y profesionales universitarios (con cinco o más años de estudios) se matriculan en las universidades que alcanzaron los mayores 
puntajes PSU. Aun más, el mayor porcentaje de este tipo de estudiantes se presenta, precisamente, en las dos instituciones que incorporan a los puntajes más altos: PUC y Universidad de Chile (donde, a su vez, se matricula la cantidad más baja de estudiantes hijos de obreros o de padres que desarrollen oficios).

\begin{tabular}{llccc}
\hline Lugar & UNIVERSIDAD & $\begin{array}{c}\text { \% Directivos/cinco } \\
\text { 0 más años }\end{array}$ & \% Empleados & \% Oficio/obrero \\
\hline 1 & Pontificia Univ. Católica de Chile & 60,9 & 30,0 & 9,1 \\
2 & De Chile & 40,5 & 45,4 & 14,2 \\
3 & De Concepción & 25,9 & 43,7 & 30,5 \\
4 & Téc. Federico Sta. María & 30,0 & 47,5 & 22,5 \\
5 & Pontifica Univ. Católica de Valparaíso & 27,3 & 49,3 & 23,4 \\
6 & De Valparaíso & 20,6 & 51,1 & 28,3 \\
7 & De Santiago & 18,8 & 50,3 & 30,9 \\
8 & Austral & 19,0 & 53,8 & 27,2 \\
9 & Católica del Norte & 20,4 & 48,8 & 30,8 \\
10 & De Antofagasta & 21,0 & 47,6 & 31,4 \\
11 & De La Frontera & 17,5 & 49,8 & 32,7 \\
12 & De Talca & 17,9 & 46,1 & 36,1 \\
13 & De La Serena & 14,5 & 46,4 & 39,2 \\
14 & Del Bío-Bío & 11,2 & 41,1 & 47,7 \\
15 & De Tarapacá & 14,2 & 50,5 & 35,3 \\
16 & Católica de la Santísima Concepción & 17,3 & 44,9 & 37,9 \\
17 & Católica de Temuco & 13,0 & 49,8 & 37,1 \\
18 & Tecnológica Metropolitana & 13,5 & 52,3 & 34,2 \\
19 & De Playa Ancha & 13,9 & 46,9 & 39,2 \\
20 & Arturo Prat & 12,4 & 45,5 & 42,1 \\
21 & Metropolitana de Ciencias de la Educ. & 14,4 & 47,7 & 37,9 \\
22 & Católica del Maule & 11,6 & 43,4 & 45,0 \\
23 & De Magallanes & 11,4 & 56,0 & 32,6 \\
24 & De Los Lagos & 8,1 & 43,6 & 48,3 \\
25 & De Atacama & 10,2 & 46,5 & 43,4 \\
\hline
\end{tabular}

La situación contraria se verifica en las universidades de Los Lagos, del Bío-Bío, de Atacama y Arturo Prat, en que la cantidad de alumnos hijos de obreros o personas con oficio se acerca al 50\%, en desmedro de estudiantes cuyos padres sean profesionales o administrativos de categoría superior. En efecto, es la misma Universidad de Los Lagos la que presenta la menor cantidad de este último tipo de estudiantes: sólo el 8,1\%. 


\section{III.12. Ocupación principal de la madre}

Para analizar la ocupación de la madre se utilizó la misma recategorización realizada para los padres. Sin embargo, y debido a que más de la mitad de los casos registraban esta característica (51\%), se optó por crear una cuarta posibilidad: "dueña de casa" (criterio que en los padres no superaba el 1\%), la cual se convierte en la más frecuente en todas las universidades, tal como se aprecia en la siguiente tabla:

\begin{tabular}{llcccc}
\hline Lugar & UNIVERSIDAD & $\begin{array}{c}\text { \% Directivas/cinco } \\
\text { o más años }\end{array}$ & \% Empleados & \% Oficio/obrero & \% Dueña de casa \\
\hline 1 & Pontificia Univ. & 30,2 & 22,7 & 3,5 & 43,6 \\
& Católica de Chile & & & & \\
2 & De Chile & 25,2 & 26,2 & 4,4 & 44,2 \\
3 & De Concepción & 17,1 & 21,8 & 6,1 & 55,0 \\
4 & De La Frontera & 16,2 & 22,6 & 6,2 & 55,0 \\
5 & Téc. Federico Sta. María & 14,9 & 22,7 & 6,4 & 55,9 \\
6 & Pontifica Univ. & 14,9 & 24,2 & 7,5 & 53,3 \\
& Católica de Valparaíso & & & & \\
7 & De Talca & 14,0 & 23,6 & 9,3 & 53,1 \\
8 & Austral & 13,7 & 27,2 & 9,1 & 50,0 \\
9 & De Valparaíso & 12,5 & 26,4 & 8,7 & 52,3 \\
10 & Católica del Norte & 11,8 & 23,4 & 5,5 & 59,3 \\
11 & Católica de Temuco & 11,2 & 22,0 & 8,4 & 58,4 \\
12 & De Antofagasta & 11,1 & 23,6 & 6,9 & 58,4 \\
13 & Católica de la Santísima & 10,9 & 22,0 & 7,3 & 59,7 \\
& Concepción & & & & \\
14 & De Santiago & 10,6 & 23,9 & 9,5 & 56,0 \\
15 & Católica del Maule & 9,5 & 15,5 & 9,7 & 65,3 \\
16 & Metropolitana de & 9,3 & 21,5 & 13,6 & 55,6 \\
& Ciencias de la Educ. & & & & \\
17 & De La Serena & 9,0 & 17,7 & 11,3 & 62,0 \\
18 & De Tarapacá & 8,4 & 19,6 & 12,6 & 59,4 \\
19 & Tecnológica Metropolitana & 8,2 & 24,5 & 12,8 & 54,5 \\
20 & Del Bío-Bío & 7,6 & 17,9 & 9,1 & 65,5 \\
21 & De Los Lagos & 7,5 & 18,4 & 12,2 & 61,8 \\
22 & De Playa Ancha & 7,5 & 24,2 & 12,6 & 55,7 \\
23 & Arturo Prat & 7,3 & 19,4 & 9,8 & 63,5 \\
24 & De Magallanes & 7,0 & 23,2 & 11,5 & 58,3 \\
25 & De Atacama & 4,9 & 20,8 & 15,0 & 59,3 \\
\hline
\end{tabular}

Al evaluar dicha categoría, es posible concluir que esta funciona prácticamente como una constante, ya que su valor es 
elevado en todas las instituciones comparadas. Ello dificulta el proceso de discriminación, pudiendo diferenciarse sólo a la PUC y a la UCH de las restantes 23 instituciones. En estas dos instituciones, menos de la mitad de sus estudiantes tiene una madre dueña de casa; aun más, sólo en ellas el porcentaje de madres con actividades de tipo administrativas superiores supera el $25 \%$, valor que disminuye a un 4,9\% para la Universidad de Atacama. No obstante, la institución que acumula la mayor cantidad de madres no es la de Atacama: es la Universidad del Bío-Bío, donde se encuentra un $65,5 \%$ de madres dueñas de casa.

\section{III.13. Situación ocupacional de ambos padres}

Finalmente, es posible caracterizar a los estudiantes que se matriculan en cada una de las universidades en función de la situación ocupacional actual de sus dos padres. Para desarrollar este análisis, y teniendo en cuenta que de las siete categorías ${ }^{v}$ que la variable inicialmente presentaba, algunas de ellas no alcanzaron una representación notoria (por ejemplo, para los hombres la categoría "dueño de casa" no superó el 0,2\%), se optó por considerar para el análisis solamente la clasificación "tiene trabajo activo remunerado", lo que se presenta en la próxima tabla:

\begin{tabular}{rlcc}
\hline & UNIVERSIDAD & $\begin{array}{c}\text { \% de padres con trabajo } \\
\text { activo remunerado }\end{array}$ & $\begin{array}{c}\text { \% de madres con trabajo } \\
\text { activo remunerado }\end{array}$ \\
\hline 1 & Pontificia Univ. Católica de Chile & 82,3 & 48,0 \\
2 & De Chile & 76,6 & 48,7 \\
3 & De Antofagasta & 74,8 & 33,4 \\
4 & Téc. Federico Sta. María & 73,7 & 37,4 \\
5 & Católica del Norte & 73,6 & 35,3 \\
6 & Pontifica Univ. Católica de Valparaíso & 71,7 & 38,7 \\
7 & De Magallanes & 69,3 & 32,2 \\
8 & De Santiago & 69,0 & 37,2 \\
9 & De Valparaíso & 68,8 & 40,9 \\
10 & De La Serena & 68,4 & 31,3 \\
11 & De Atacama & 68,3 & 32,5 \\
12 & Austral & 68,0 & 43,4
\end{tabular}

v Activo (tiene trabajo remunerado); Trabaja sólo ocasionalmente; Cesante, Jubilado; No trabaja, Dueña de casa; otro. 
13 De Concepción

14 Tecnológica Metropolitana

15 De Playa Ancha

16 Metropolitana de Ciencias de la Educ.

17 Católica de la Santísima Concepción

18 Católica del Maule

19 De Talca

20 De La Frontera

21 Católica de Temuco

22 Arturo Prat

23 Del Bío-Bío

24 De Los Lagos

25 De Tarapacá
67,9

67,7

66,3

66,1

66,1

65,9

65,6

64,2

63,3

61,3

57,7

57,5

55,2
38,6

36,3

36,4

Al observar la tabla, se concluye que, independiente de la universidad que se desea analizar, más de la mitad de los padres posee trabajo activo remunerado, pero menos del 50\% de las madres registra igual situación (la mayoría de las madres fue calificada como "dueña de casa", actividad no remunerada, lo que da cuenta de esta diferencia).

Asimismo, en determinadas instituciones el porcentaje de padres con trabajo remunerado supera el $70 \%$, siendo la PUC el lugar donde se encuentra la mayor cantidad de estudiantes matriculados cuyo padre posee un trabajo activo (lo que coincide con el mayor porcentaje de madres con trabajo remunerado). Esta afirmación es igualmente aplicable a las universidades de Chile, de Antofagasta, Técnica Federico Santa María, del Norte y de Valparaíso.

Se concluye, entonces, que el hecho de que el padre y/o la madre tengan un trabajo activo con compensación económica se relaciona con la elección de un centro universitario específico. Efectivamente, los estudiantes cuyos padres y/o madres presentan un trabajo remunerado tienden a matricularse en las universidades de puntajes PSU más altos (como la PUC, la UCH y la UTFSM). La gran excepción la constituye la Universidad de Antofagasta, la cual, pese a inscribir alumnos con padre y madre laboralmente activos, sólo posee un promedio PSU de 547 puntos. 


\section{III.14. Índice de preferencia}

Resulta interesante observar a las universidades en su conjunto; es decir, compararlas en términos del tipo de estudiante que preferentemente la elige como casa de estudio. Para ello se han seleccionado las variables sociodemográficas y educacionales y han sido trabajadas de manera comparativa para elaborar un índice de preferencia de los estudiantes que, egresando de educación media en 2005, se han matriculado en una de las 25 universidades del Consejo de Rectores. Esta actividad permite conocer cuáles son las características principales de los estudiantes y a qué universidades postulan y se matriculan; dicho de otra manera, cómo responden estas instituciones de acuerdo al perfil de los estudiantes.

Como una manera de comparar a las veinticinco casas de estudio, se ingresó en una tabla el lugar relativo de cada universidad para cada una de las variables. Así, a las instituciones se les puede asignar un valor que fluctúa entre 1 y 25 en cada una de las variables. Para obtener el índice descrito se procedió a promediar los valores que cada universidad obtiene. El promedio entrega un valor que va entre 1 y 25 , siendo el valor 1 el valor más alto y el 25 el más bajo. Por ejemplo, aquella universidad que, dentro de su población de estudiantes, presente el mayor porcentaje de alumnos egresados de establecimientos particulares pagados se le asignará el valor "1" en dicho atributo. Por el contrario, aquella casa de estudio que presente la menor proporción de estudiantes egresados de dichos establecimientos tendrá el valor "25".

Además, se incorpora la desviación estándar (DS), medida que permite observar el grado de homogeneidad de cada universidad en relación a las variables analizadas. Es decir, permite determinar si la institución posee variaciones en relación a su propio promedio. De este modo, las instituciones que obtengan una desviación estándar pequeña, serán aquellas que no presenten grandes diferencias en su posición ante cada uno de los criterios considerados. 
Las variables que se consideraron para construir el índice (y su sigla correspondiente) son las siguientes: porcentaje de estudiantes que reportan ingresos familiares superiores a $\$ 2.500 .000$ (\% > IF); porcentaje de alumnos egresados de establecimientos particulares pagados $(\%>$ PP); porcentaje de estudiantes que egresaron de establecimientos humanista-científico $(\%>\mathrm{HC})$; porcentaje de estudiantes cuyo padre presente niveles universitarios de educación (Educ. padre); porcentaje de estudiantes cuya madre presente niveles universitarios de educación (Educ. madre); porcentaje de estudiantes cuyos padres ocupan cargos de gerentes o de profesionales (Ocup. padre); porcentaje de estudiantes cuyas madres ocupan cargos de gerentes o de profesionales (Ocup. madre); porcentaje de alumnos que postulan a la casa de estudios dentro de su tres preferencias (Postula.); puntaje promedio PSU de la casa de estudios y, por último, el valor correspondiente en el índice de preferencia.

Se debe tener en cuenta que siete de las variables pueden ser consideradas características pertenecientes al estudiante, previas al proceso de rendición de la PSU. El porcentaje de preferencias a cada universidad y el puntaje promedio que alcanzan los estudiantes que se matriculan en ellas, en cambio, se obtienen una vez finalizada la rendición de la PSU. A continuación se presenta la tabla con los resultados:

\begin{tabular}{|c|c|c|c|c|c|c|c|c|c|c|c|}
\hline UNIVERSIDAD & $\%>I F$ & $\begin{array}{l}\% \text { de } \\
>\mathrm{PP} \\
\end{array}$ & $\begin{array}{c}\% \mathrm{de} \\
\mathrm{HC}\end{array}$ & $\begin{array}{l}\text { Educ. } \\
\text { padre }\end{array}$ & $\begin{array}{l}\text { Educ. } \\
\text { madre }\end{array}$ & $\begin{array}{l}\text { Ocup. } \\
\text { padre }\end{array}$ & $\begin{array}{l}\text { Ocup. } \\
\text { madre }\end{array}$ & Postula & $\begin{array}{c}\text { Puntaje } \\
\text { PSU }\end{array}$ & Índice & DS \\
\hline $\begin{array}{l}\text { Pontificia Univ. } \\
\text { Católica de Chile }\end{array}$ & l & 1 & 1 & 1 & 1 & 1 & 1 & 3 & 1 & 1,2 & 0,67 \\
\hline De Chile & 2 & 2 & 2 & 2 & 2 & 2 & 2 & 1 & 2 & 1,9 & 0,33 \\
\hline Téc. Federico Sta. María & 3 & 3 & 9 & 3 & 3 & 4 & 5 & 7 & 3 & 4,4 & 2,19 \\
\hline $\begin{array}{l}\text { Pontifica Univ. } \\
\text { Católica de Valparaíso }\end{array}$ & 4 & 4 & 3 & 4 & 5 & 5 & 6 & 6 & 5 & 4,7 & 1 \\
\hline De Concepción & 6 & 6 & 12 & 5 & 4 & 3 & 3 & 2 & 6 & 5,2 & 2,95 \\
\hline De Valparaíso & 9 & 5 & 5 & 8 & 9 & 6 & 9 & 4 & 11 & 7,3 & 2,4 \\
\hline De La Frontera & 8 & 14 & 8 & 10 & 6 & 11 & 4 & 8 & 7 & 8,4 & 2,92 \\
\hline Austral & 10 & 10 & 4 & 9 & 7 & 8 & 8 & 10 & 12 & 8,7 & 2,29 \\
\hline De Santiago & 12 & 9 & 10 & 11 & 12 & 7 & 14 & 5 & 4 & 9,3 & 3,39 \\
\hline Católica del Norte & 7 & 7 & 14 & 7 & 10 & 9 & 10 & 13 & 13 & 10 & 2,78 \\
\hline De Talca & 13 & 12 & 19 & 13 & 8 & 12 & 7 & 14 & 9 & 11,9 & 3,62 \\
\hline De Antofagasta & 5 & 8 & 18 & 6 & 11 & 10 & 12 & 18 & 19 & 11,9 & 5,33 \\
\hline Católica de la & 11 & 11 & 17 & 12 & 14 & 16 & 13 & 17 & 17 & 14,2 & 2,59 \\
\hline
\end{tabular}




\begin{tabular}{lccccccccccc}
$\begin{array}{l}\text { Metropolitana de } \\
\text { Ciencias de la Educ. }\end{array}$ & 14 & 15 & 16 & 18 & 16 & 21 & 16 & 16 & 8 & 15,6 & 3,47 \\
De Playa Ancha & 16 & 16 & 6 & 15 & 19 & 19 & 22 & 11 & 18 & 15,8 & 4,79 \\
Católica de Temuco & 15 & 20 & 7 & 20 & 13 & 17 & 11 & 19 & 21 & 15,9 & 4,78 \\
De La Serena & 22 & 23 & 11 & 17 & 17 & 13 & 17 & 9 & 15 & 16 & 4,64 \\
De Tarapacá & 21 & 18 & 15 & 16 & 15 & 15 & 18 & 15 & 22 & 17,2 & 2,73 \\
Católica del Maule & 18 & 17 & 13 & 24 & 18 & 22 & 15 & 22 & 14 & 18,1 & 3,86 \\
Del Bío-Bío & 17 & 19 & 24 & 23 & 24 & 14 & 20 & 12 & 16 & 18,8 & 4,38 \\
Tecnológica Metropolitana & 20 & 21 & 22 & 19 & 20 & 18 & 19 & 21 & 10 & 18,9 & 3,55 \\
Arturo Prat & 19 & 25 & 23 & 14 & 21 & 20 & 23 & 20 & 23 & 20,9 & 3,22 \\
De Magallanes & 23 & 13 & 20 & 22 & 22 & 23 & 24 & 24 & 20 & 21,2 & 3,42 \\
De Los Lagos & 23 & 22 & 21 & 25 & 23 & 24 & 21 & 23 & 25 & 23 & 1,5 \\
De Atacama & 25 & 24 & 25 & 21 & 25 & 25 & 25 & 25 & 24 & 24,3 & 1,32 \\
\hline & & & & & & & & & & &
\end{tabular}

Al revisar los valores promedios de cada institución, se determina que tanto la PUC como la UCH, debido a que obtienen promedios cercanos a uno (1,2 y 1,9 respectivamente) presentan estudiantes matriculados con las variables estudiadas en su máxima expresión. Asimismo, se debe destacar la baja desviación estándar de esas instituciones, lo que implica un comportamiento homogéneo en las diferentes características. En otras palabras, ambas universidades reciben estudiantes con un perfil claro: mayoritariamente egresados de establecimientos científico-humanistas, que alcanzan los más altos puntajes en la PSU, con ambos padres de altos niveles educacionales (universitario) y que ocupan cargos gerenciales o profesionales, que les permiten reportar ingresos familiares altos. En este mismo sentido, las universidades se ordenan en función de la preferencia, obteniendo alguna de ellas valores altos (superiores a 20, situación propia a las universidades de Magallanes, Arturo Prat, Los Lagos y Atacama), lo que indica que son corporaciones, en general, cuyos estudiantes tienen condiciones socioeconómicas vulnerables y, también, que obtienen los puntajes más bajos en la PSU (del grupo de alumnos que finalmente se matricula).

Otro aspecto que se puede destacar es que las seis universidades con el más alto índice de preferencia se concentran exclusivamente en tres regiones del país, a saber: Metropolitana, V y VIII.

Asimismo, es posible afirmar que, debido a su alta desviación estándar, las Universidades de Antofagasta ( $S=5.33$ ), Playa Ancha 
( $\mathrm{S}=4.79)$, Católica de Temuco (4.78) y de La Serena ( $\mathrm{S}=4.64)$ resultan ser las que ingresan estudiantes con mayores diferencias entre ellos (grupos más heterogéneos).

\section{Conclusiones}

El estudio realizado permite afirmar que el creciente acceso a la educación superior presenta diferencias según grupos socioeconómicos.

En lo que respecta a la evolución de la población en estudio, desde el proceso de rendir la PSU hasta el de matricularse, es posible afirmar que son los sectores más desfavorecidos en términos socioeconómicos (aquellos que provienen de sectores con menos ingresos familiares, cuyos padres poseen menores niveles educacionales y trabajos menos remunerados) y que han estudiado en colegios prioritariamente subvencionados y técnico-profesionales, quienes, proporcionalmente, tienen menos oportunidades de llegar a alguna de las universidades del Consejo de Rectores.

La afirmación anterior se ratifica cuando se analizan las características de la submuestra de quienes no postulan (teniendo puntaje para ello), y se comparan con quienes sí lo hacen. La tendencia se repite: aquellos que no postulan proceden, proporcionalmente, más de sectores desfavorecidos en términos socioeconómicos y educacionales. Puede decirse entonces que la condición socioeconómica y educacional se relaciona con los procesos de postulación e ingreso a una universidad del Consejo de Rectores. Dicha relación es directa por lo que mejorarán las probabilidades de postulación e ingreso en la medida en que se tengan mejores condiciones sociales y económicas, y, también, se provenga de colegios particulares pagados y científico-humanistas.

Dos de las variables descritas requieren una mención especial.

La primera dice relación con los puntajes obtenidos en la PSU. El puntaje puede ser interpretado, por una parte, como variable de 
resultado de las variables de caracterización y, por otra, como variable interviniente en el proceso de postulación y matrícula. Si la observamos como resultado, podemos afirmar que los estudiantes de mayor vulnerabilidad social y educativa obtienen menores puntajes en la PSU, tal como se pudo observar en los resultados de este estudio. Por otra parte, al observar los puntajes de la PSU como una variable interviniente para el proceso de postulación y matrícula, se puede afirmar que, para el caso de la postulación, existe una correlación positiva entre postulación y puntaje en la PSU. Para los efectos de matrícula es posible afirmar que los puntajes promedio de matrícula tenderán a crecer en relación a los de postulación para cada universidad.

La segunda variable de refiere al sexo de los estudiantes. Puede afirmarse que, aunque más mujeres rinden la PSU, serán menos las que se matriculen. Lo anterior indica que la condición de sexo es un variable asociada al proceso de matrícula a una universidad.

Puede afirmarse, entonces, que la probabilidad de ingresar a una universidad del Consejo de Rectores estará relacionada diferencialmente de acuerdo con un diferente comportamiento de las variables de estratificación.

Por otra parte, al intentar relacionar la oferta de las universidades y el interés de los alumnos por determinadas instituciones, se puede afirmar que las universidades se estratifican en función de las características de los estudiantes. Al respecto, es posible señalar que el perfil del alumno es diferente entre una universidad y otra o, dicho de otra manera, que existe un perfil de alumno por universidad. Ello se ratifica al observar el índice de preferencia en el que se consideraron las variables que más estratifican.

En síntesis, las universidades congregan a estudiantes que tienen características diferentes en términos sociodemográficos y educacionales. La pregunta que surge es si son las universidades las que seleccionan a los estudiantes, si son éstos quienes lo hacen con las universidades o si lo que se produce es una interacción 
entre las características de unos y otras. En este sentido, es relevante desarrollar otros estudios que permitan dilucidar cuáles son los aspectos que son tomados en cuenta por los estudiantes al momento de elegir una universidad y cómo éstos interactúan con las características reales o percibidas de las propias instituciones, con el fin de poder verificar la hipótesis de este estudio, a saber, que la variable socioeconómica y/o cultural-valórica tiene relevancia al momento de elegir la institución formadora.

Por lo pronto, se puede afirmar que la elección de la universidad esta relacionada con un conjunto de variables tanto sociodemográficas como educacionales.

\section{Referencias bibliográficas}

Belleï, C. (2003) ¿Ha tenido impacto la reforma educativa chilena? En: Cox C, editor. Políticas educacionales en el cambio de siglo. Santiago de Chile: Editorial Universitaria.

Brown, R.J. (1997) Prejudice: its social psychology. Oxford: Blackwell.

Fiske, S. (2000) Controlling other People: The impact of power on stereotyping. American Psychology Association, 48, (6), pp. 621-628.

Hogg, M. \& Abrams, D. (1988) Social identifications. London: Routledge.

Lemaitre, M.J. (2003) La reforma de la educación media. En: Cox C, editor. Políticas educacionales en el cambio de siglo. Santiago de Chile: Editorial Universitaria.

Morales, J. F; Páez, D.; Deschamps, J.C. y Worchel, S., editores. (1996) Identidad social. Valencia: Promolibro, pp. 255-285.

Shaw, M. (1986) Dinámica de grupos. Barcelona: Herder.

Tajfel, H. (1984) Grupos humanos y categorías sociales. Barcelona: Herder.

Turner, J. (1990) Redescubrir el grupo social. Madrid: Morata.

Recibido: 16 de mayo de 2006

Aceptado: 30 de mayo de 2006 